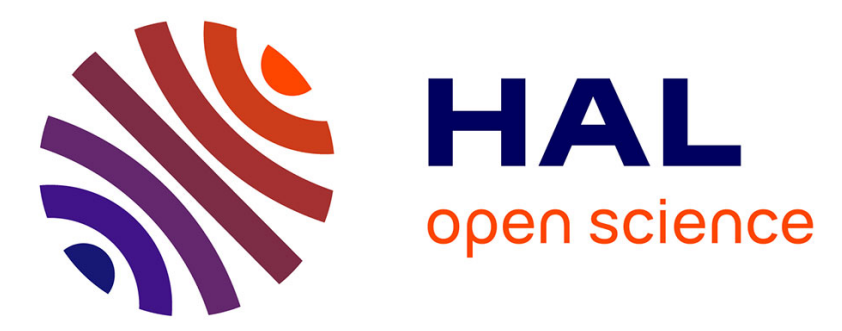

\title{
BEC2HPC: a HPC spectral solver for nonlinear Schrödinger and Gross-Pitaevskii equations. Stationary states computation
}

Jérémie Gaidamour, Qinglin Tang, Xavier Antoine

\section{- To cite this version:}

Jérémie Gaidamour, Qinglin Tang, Xavier Antoine. BEC2HPC: a HPC spectral solver for nonlinear Schrödinger and Gross-Pitaevskii equations. Stationary states computation. Computer Physics Communications, 2021, 265, 10.1016/j.cpc.2021.108007 . hal-02957115

\section{HAL Id: hal-02957115 \\ https://hal.science/hal-02957115}

Submitted on 4 Oct 2020

HAL is a multi-disciplinary open access archive for the deposit and dissemination of scientific research documents, whether they are published or not. The documents may come from teaching and research institutions in France or abroad, or from public or private research centers.
L'archive ouverte pluridisciplinaire HAL, est destinée au dépôt et à la diffusion de documents scientifiques de niveau recherche, publiés ou non, émanant des établissements d'enseignement et de recherche français ou étrangers, des laboratoires publics ou privés. 


\title{
BEC2HPC: a HPC spectral solver for nonlinear Schrödinger and Gross-Pitaevskii equations. Stationary states computation.
}

\author{
Jérémie GAIDAMOUR ${ }^{\mathrm{a}}$, Qinglin TANG ${ }^{\mathrm{b}}$, Xavier ANTOINE ${ }^{\mathrm{a}}$ \\ ${ }^{a}$ Université de Lorraine, CNRS, Inria, IECL, F-54000 Nancy, France. \\ ${ }^{b}$ School of Mathematics, State Key Laboratory of Hydraulics and Mountain River Engineering, Sichuan University, \\ Chengdu 610064, China.
}

\begin{abstract}
We present BEC2HPC which is a parallel HPC spectral solver for computing the ground states of the nonlinear Schrödinger equation and the Gross-Pitaevskii equation (GPE) modeling rotating BoseEinstein condensates (BEC). Considering a standard pseudo-spectral discretization based on Fast Fourier Transforms (FFTs), the method consists in finding the numerical solution of the energy functional minimization problem under normalization constraint by using a preconditioned nonlinear conjugate gradient method. We present some numerical simulations and scalability results for the 2D and 3D problems to obtain the stationary states of BEC with fast rotation and large nonlinearities. The code takes advantage of existing HPC libraries and can itself be leveraged to implement other numerical methods like e.g. for the dynamics of BECs.
\end{abstract}

Keywords: Bose-Einstein condensation; nonlinear Schrödinger equation; Gross-Pitaevskii equation; stationary states; pseudo-spectral method; nonlinear conjugate gradient; high performance computing

\section{Introduction}

Bose-Einstein Condensates (BECs) have been first predicted theoretically by S.N. Bose and A. Einstein, before their experimental realization in 1995 [5, 23, 30,34]. This state of matter has the interesting feature that macroscopic quantum physics properties can emerge and be observed in a laboratory experiment. The literature on BECs grown very fast over the last two decades in atomic, molecular, optics and condensed matter physics. Important applications related to this new physics are now appearing, like e.g. in quantum computing [25]. Concerning the most important directions, a special attention has been directed towards the understanding of the nucleation of vortices [1, 24, 48, 49,51,55, 63], properties of dipolar gases $[17,18]$ or also multi-components BECs $[15,16,17]$. For temperatures $T$ which are much smaller than the critical temperature $T_{c}$, the macroscopic behavior of a BEC can be correctly described by a condensate wave function $\psi$ as the solution to a Gross-Pitaevskii Equation (GPE). Therefore, being able to numerically calculate efficiently the solution of such a class of equations is extremely useful. Concerning the main questions related to BECs, we can cite the calculation of the stationary states (ground/excited states) as well as the real-time dynamics [6, 10, 17, 38, 43].

In the present paper, we consider the problem of the computation of stationary states (most particularly ground states) of the rotating GPE. A few methods are available in the literature to numerically obtain them. For example, some algorithms are based on appropriate discretizations of the continuous normalized gradient flow/imaginary-time formulation [3, 8, 10, 17, 19, 22, 28, 29, 64], leading to various iterative algorithms. The methods are general and can be directly extended to many physical situations (e.g. dipolar interactions, multi-components GPEs...) [7, 8, 10, 17, 19]. Other approaches rather solve numerically the nonlinear eigenvalue problem $[35,60]$ or the minimization of the energy functional by using optimization techniques under constraints [21, 26, 31, 32, 33]. Regularized Newton-type methods can also be used [61]. In the present paper, we consider the constrained nonlinear Preconditioned Conjugate Gradient (PCG) method with a pseudo-spectral discretization scheme for the rotating GPE. This

Email addresses: jeremie.gaidamour@univ-lorraine.fr (Jérémie GAIDAMOUR), qinglin_tang@163.com (Qinglin TANG), xavier.antoine@univ-lorraine.fr (Xavier ANTOINE)

URL: http://iecl.univ-lorraine.fr/ xantoine/ (Xavier ANTOINE) 
approach, developed in [11] and presented shortly in Section 2, provides an efficient and robust way to solve the minimization problem. Even if only the rotating GPE is considered here, the method can be extended to other situations (see [14] for dipole-dipole interactions or [12, 13] for nonlinear fractional GPEs). The main goal of the paper is to introduce the solver BEC2HPC, to explain how to use its functionalities and to provide a few examples. Some future developments will include general systems of GPEs, as well as additional numerical methods for the dynamics of the GPE. Finally, let us remark that the GPE can be simplified to the nonlinear Schrödinger equation. Therefore, the BEC2HPC solver can also be useful to simulate other physical situations than BEC, where the Schrödinger equation has to be numerically solved efficiently and accurately.

Concerning the available solvers for the GPE (and nonlinear Schrödinger equation), several contributions exist in the literature. Adhikari, Muruganandam and their co-authors developed in a series of papers some finite-difference codes for computing the stationary states and dynamics of GPEs without rotation. Various implementations are available, including codes written in Fortran [45, 52], C [45, 58], with OpenMP/MPI/CUDA/GNU versions [47, 56, 62]. In addition, the contribution [44] proposes a code that can solve the GPE with rotation term. In [42], a Matlab toolbox called OCTBEC is designed for the optimal control of BECs. The GPU-accelerated Matlab/C toolbox NLSEmagic is developed in [27] for solving the multi-dimensional nonlinear Schrödinger equation through finite-difference in space and with a fourth-order Runge-Kutta scheme in time. In [50], a finite element C++ toolbox is proposed for computing the stationary states (based on a Newton method) and dynamics of nonlinear Schrödinger equations. Another finite element toolbox has been recently developed with Freefem ++ for solving various problems related to the 2D and 3D rotating GPE [57]. Finally, GPELab [7, 9] is a Matlab toolbox that can solve a large class of problems related to the GPE, including stationary states, dynamics, and the possibility to handle some additional stochastic terms. The wide variety of problems which can be solved by GPELab leads to the possibility of simulating many complex physical configurations related to BECs, which makes the toolbox very attractive. For the stationary states, the solver used in GPELab may sometimes converge slowly for large nonlinearities and high rotation speeds, since i) it is based on the normalized gradient flow/imaginary time, and ii) the implementation in Matlab does not permit to use optimally the HPC ressources. In BEC2HPC, we use the PCG algorithm which is known to outperfom the normalized gradient flow formulation as shown in [11]. In addition, BEC2HPC proposes a HPC implementation of the algorithm, for the 2D and 3D GPE with rotation term. The resulting solver is then very robust, efficient and highly accurate since it uses a pseudo-spectral approximation in space.

The plan of the paper is the following. In Section 2, we introduce the PCG algorithm that is used in BEC2HPC for computing the stationary states. Section 3 gives some informations about the way BEC2HPC is developed, most particularly regarding the FFT implementation and some parallelization aspects. In Section 4, we propose a relatively simple example to start with the use of BEC2HPC, after its installation. Section 5 is devoted to the most advanced features of BEC2HPC, including the data definition and the parameter selection, the use of the visualization tool (ParaView). In Section 6, we provide more examples and report some performances of the solver in 2D and 3D. Finally, we conclude in Section 7.

\section{The PCG method for computing stationary states of the GPE}

\subsection{Notations and formulation}

Let us consider the problem of computing a ground state of a $d$-dimensional $(d=2,3)$ BEC which can be written under the form of the constrained minimization problem

$$
\left\{\begin{array}{l}
\text { Find } \phi \in L^{2}\left(\mathbb{R}^{d}\right) \text { such that } \\
\phi \in \arg \min _{\|\phi\|_{2}=1} \mathcal{E}_{\text {tot }}(\phi),
\end{array}\right.
$$

where the $L^{2}\left(\mathbb{R}^{d}\right)$-norm of $\phi$ is defined as

$$
\|\phi\|_{2}^{2}=\int_{\mathbb{R}^{d}}|\phi|^{2} d \mathbf{x}:=<\phi, \phi>
$$

and the hermitian inner-product is

$$
\forall(u, v) \in L^{2}\left(\mathbb{R}^{d}\right) \times L^{2}\left(\mathbb{R}^{d}\right), \quad\left\langle u, v>:=\int_{\mathbb{R}^{d}} u v^{*} d \mathbf{x},\right.
$$


definining $v^{*}$ as the complex conjugate of $v$. For the minimization problem (2.1), we introduce the total energy functional $\mathcal{E}_{\text {tot }}$ for the dimensionless rotating GPE defined by

$$
\begin{aligned}
\mathcal{E}_{\text {tot }}(\phi) & =\int_{\mathbb{R}^{d}}\left[\frac{1}{2}|\nabla \phi|^{2}+V(\mathbf{x})|\phi|^{2}+F\left(|\phi|^{2}\right)-\operatorname{Re}\left(\phi^{*} \boldsymbol{\Omega} \cdot \mathbf{L} \phi\right)\right] d \mathbf{x} \\
& :=\mathcal{E}_{\text {kin }}(\phi)+\mathcal{E}_{\text {pot }}(\phi)+\mathcal{E}_{\text {int }}(\phi)+\mathcal{E}_{\text {rot }}(\phi),
\end{aligned}
$$

for $t>0$. In 3D, the Laplace operator is given by: $\Delta=\nabla^{2}$, where $\nabla:=\left(\partial_{x}, \partial_{y}, \partial_{z}\right)^{t}$ is the gradient operator; the spatial variable is $\mathbf{x}=(x, y, z)^{t} \in \mathbb{R}^{3}$ (in $2 \mathrm{D}$, we have $\nabla:=\left(\partial_{x}, \partial_{y}\right)^{t}$ and $\mathbf{x}=(x, y)^{t} \in \mathbb{R}^{2}$ ). The function $V$ represents the external (usually confining) potential. The smooth real-valued function $f(\rho):=F^{\prime}(\rho)$ models the nonlinearity, setting $\rho=|\phi|^{2}$ as the density function. A first example consists in the standard cubic case which reads as

$$
F(\rho)=\beta \rho^{2} / 2,
$$

and then $f(\rho)=\beta \rho$, where $\beta$ is the nonlinearity strength describing the interaction between atoms of the condensate. This parameter is related to the $s$-scattering length $\left(a_{s}\right)$ and is positive (respectively negative) for a repulsive (respectively attractive) interaction. Other kinds of nonlinearities involve e.g. nonlocal nonlinear interactions like the dipole-dipole interaction [10, 12, 13, 14, 20]. For vortices creation, a rotating term is added. The vector $\boldsymbol{\Omega}$ is the angular velocity vector and the angular momentum is $\mathbf{L}=\left(L_{x}, L_{y}, L_{z}\right)=\mathbf{x} \wedge \mathbf{P}$, with the momentum operator $\mathbf{P}=-i \nabla$. In many situations, the angular velocity is such that $\boldsymbol{\Omega}=(0,0, \omega)^{t}$ leading to

$$
\mathbf{\Omega} \cdot \mathbf{L}=\omega \mathcal{L}_{z}=-i \omega\left(x \partial_{y}-y \partial_{x}\right)
$$

A direct computation of the energy gradient yields

$$
\nabla \mathcal{E}_{\text {tot }}(\phi)=2 \mathcal{H}_{\phi} \phi, \quad \text { with } \quad \mathcal{H}_{\phi}=-\frac{1}{2} \Delta+V+f\left(|\phi|^{2}\right)-\boldsymbol{\Omega} \cdot \mathbf{L}
$$

and the second-order derivative is

$$
\frac{1}{2} \nabla^{2} \mathcal{E}_{\text {tot }}(\phi)[u, u]=\left\langle u, \mathcal{H}_{\phi} u\right\rangle+\operatorname{Re}\left\langle f\left(\phi^{2}\right), u^{2}\right\rangle .
$$

Let us introduce $\mathcal{S}=\left\{\phi \in L^{2}\left(\mathbb{R}^{d}\right),\|\phi\|_{2}=1\right\}$ as the unit spherical manifold associated to the normalization constraint. The tangent space at a point $\phi \in \mathcal{S}$ is given by $T_{\phi} \mathcal{S}=\left\{h \in L^{2}\left(\mathbb{R}^{d}\right), \operatorname{Re}\langle\phi, h\rangle=0\right\}$, and the orthogonal projection $M_{\phi}$ onto this space is such that $M_{\phi} h=h-\operatorname{Re}\langle\phi, h\rangle \phi$. Writing the Euler-Lagrange equation (first-order necessary condition for the minimum) associated with our problem at a minimum $\phi \in \mathcal{S}$ requires that the projection of the gradient on the tangent space $\mathcal{S}$ is zero, i.e. $M_{\phi} \nabla \mathcal{E}_{\text {tot }}(\phi)=0$. This equation is equivalent to the nonlinear eigenvalue problem

$$
\mathcal{H}_{\phi} \phi=\lambda \phi
$$

where $\lambda:=\lambda(\phi)=\left\langle\mathcal{H}_{\phi} \phi, \phi\right\rangle$ is the Lagrange multiplier associated to the spherical constraint (also known as the chemical potential).

\subsection{Pseudospectral spatial discretization}

To find a numerical solution of the minimization problem, the function $\phi \in L^{2}\left(\mathbb{R}^{d}\right)$ must be discretized carefully and accurately, most particularly to describe fine details like the vortex lattice structure that can appear. BEC2HPC considers a standard pseudo-spectral discretization scheme based on Fast Fourier Transforms (FFTs) $[8,10,19,64]$. In 3D, we truncate the wave function $\phi$ to a square domain $\left[-a_{x}, a_{x}\right] \times$ $\left[-a_{y}, a_{y}\right] \times\left[-a_{z}, a_{z}\right]\left(a_{x}, a_{y}\right.$ and $a_{z}$ being positive), with periodic boundary conditions, and discretize $\phi$ with an even number of $n_{x}, n_{y}$ and $n_{z}$ grid points in the respective $x$-, $y$ - and $z$-directions. We describe our scheme in $3 \mathrm{D}$ (the $2 \mathrm{D}$ case being then straightforward). For $M:=\left(n_{x}, n_{y}, n_{z}\right)$, we introduce a uniformly sampled grid: $\mathcal{D}_{M}:=\left\{\mathbf{x}_{k_{1}, k_{2}, k_{3}}=\left(x_{k_{1}}, y_{k_{2}}, z_{k_{3}}\right)\right\}_{\left(k_{1}, k_{2}, k_{3}\right) \in \mathcal{O}_{M}}$, with $\mathcal{O}_{M}:=\left\{0, \ldots, n_{x}-\right.$ $1\} \times\left\{0, \ldots, n_{y}-1\right\} \times\left\{0, \ldots, n_{z}-1\right\}, x_{k_{1}+1}-x_{k_{1}}=h_{x}, y_{k_{2}+1}-y_{k_{2}}=h_{y}$ and $z_{k_{3}+1}-z_{k_{3}}=h_{z}$, with mesh sizes $h_{x}=2 a_{x} / n_{x}, h_{y}=2 a_{y} / n_{y}$ and $h_{z}=2 a_{z} / n_{z}$. By considering the $N \times N$ Hermitian 
matrix(-free) operators from $\mathbb{C}^{N}\left(N=n_{x} n_{y} n_{z}\right.$ in $\left.3 \mathrm{D}\right)$ to $\mathbb{C}$ given by $\llbracket \Delta \rrbracket:=\llbracket \partial_{x}^{2} \rrbracket+\llbracket \partial_{y}^{2} \rrbracket+\llbracket \partial_{z}^{2} \rrbracket$ and $\llbracket \boldsymbol{\Omega} \cdot \mathbf{L} \rrbracket:=-i \boldsymbol{\Omega} \cdot(\mathbf{x} \wedge \llbracket \nabla \rrbracket)$, we obtain the discretization of the gradient of the energy

$$
\left(\llbracket \nabla \rrbracket E_{\mathrm{tot}}\right)(\phi)=2 \llbracket \mathcal{H}_{\phi} \rrbracket \phi,
$$

with

$$
\llbracket \mathcal{H}_{\phi} \rrbracket:=-\frac{1}{2} \llbracket \Delta \rrbracket+\llbracket V \rrbracket+\llbracket f\left(|\phi|^{2}\right) \rrbracket-\llbracket \boldsymbol{\Omega} \cdot \mathbf{L} \rrbracket .
$$

We set $\phi:=\left(\tilde{\phi}\left(\mathbf{x}_{k_{1}, k_{2}, k_{3}}\right)\right)_{\left(k_{1}, k_{2}, k_{3}\right) \in \mathcal{O}_{M}}$ (where $\tilde{\phi}$ is the approximation of the function $\phi$ ) as the discrete unknown vector in $\mathbb{C}^{N}$. For conciseness, we identify an array $\phi$ in the vector space of $3 \mathrm{D}$ complex-valued arrays $\mathcal{M}_{n_{x}, n_{y}, n_{z}}(\mathbb{C})$ (storage according to the $3 \mathrm{D}$ grid) and the reshaped vector $\phi$ in $\mathbb{C}^{N}$. In addition, to simplify the notations, we forget the brackets $\llbracket A \rrbracket$ and use $A$ to designate the matrix operator associated with a continuous operator $A$, based on the FFT approximation. Finally, the cost for evaluating the application of a 3D FFT is $\mathcal{O}(N \log N)$.

For $\phi \in \mathbb{C}^{N}$, the total discrete energy $E_{\text {tot }}(\phi)$ can be written as the sum of the four elementary energies

$$
E_{\mathrm{tot}}(\phi)=E_{\mathrm{kin}}(\phi)+E_{\mathrm{pot}}(\phi)+E_{\mathrm{int}}(\phi)+E_{\mathrm{rot}}(\phi),
$$

setting

$$
\begin{aligned}
& E_{\text {kin }}(\phi):=\frac{1}{2}\|\nabla \phi\|_{2}^{2}=\frac{1}{2}\langle\nabla \phi, \nabla \phi\rangle=\frac{1}{2}\left(\left\langle\partial_{x} \phi, \partial_{x} \phi\right\rangle+\left\langle\partial_{y} \phi, \partial_{y} \phi\right\rangle+\left\langle\partial_{z} \phi, \partial_{z} \phi\right\rangle\right), \\
& E_{\text {pot }}(\phi):=\langle V \phi, \phi\rangle, \quad E_{\text {int }}(\phi):=\langle F(\boldsymbol{\rho}), \mathbf{1}\rangle, \quad E_{\mathrm{rot}}(\boldsymbol{\phi})=-\operatorname{Re}(\langle\boldsymbol{\Omega} \cdot \mathbf{L} \boldsymbol{\phi}, \boldsymbol{\phi}\rangle),
\end{aligned}
$$

where $\mathbf{1} \in \mathbb{C}^{N}$ is the vector with components 1 , and $\|\phi\|_{2}$ is the discretization of the $L^{2}\left(\mathbb{R}^{d}\right)$-norm on the uniform grid subject to the discrete hermitian inner product $\langle\mathbf{u}, \mathbf{v}\rangle$ for two complex-valued functions defined on the grid $\mathcal{D}_{M}$.

\subsection{The Preconditioned Conjugate Gradient (PCG) method}

Based on the pseudospectral discretization, we now need to compute the solution to the finitedimensional minimization problem under normalization constraint

$$
\phi \in \underset{\phi \in \mathbb{C}^{N},\|\boldsymbol{\phi}\|_{2}=1}{\arg \min } E_{\text {tot }}(\boldsymbol{\phi}) .
$$

In BEC2HPC, we use the Preconditioned Conjugate Gradient (PCG) method which differs from the preconditioned gradient method by the following update rule for the descent method

$$
\mathbf{d}_{n}=-P \mathbf{r}_{n}+\beta_{n} \mathbf{p}_{n-1},
$$

where $P$ designates a well-designed preconditioner and the residual vector is $\mathbf{r}_{n}:=\left(\mathcal{H}_{\phi_{n}}-\lambda_{n} I\right) \phi_{n}$. The vector $\phi_{n}$ is the iterate of $\phi$ at step $n$ for the minimization method (a preconditioned descent algorithm would lead to the relation $\mathbf{d}_{n}=-P \mathbf{r}_{n}$ ). In addition, we define $\mathbf{p}_{n}=\mathbf{d}_{n}-\operatorname{Re}\left\langle\mathbf{d}_{n}, \boldsymbol{\phi}_{n}\right\rangle \boldsymbol{\phi}_{n}$ as the orthogonal projection of $\mathbf{d}_{n}$ onto the space generated by $\phi_{n}$. The step $\beta_{n}$ is given by the Polak-Ribière formula $\beta=\max \left(\beta^{\mathrm{PR}}, 0\right)$, setting

$$
\beta^{\mathrm{PR}}=\frac{\left\langle\mathbf{r}_{n}-\mathbf{r}_{n-1}, P \mathbf{r}_{n}\right\rangle}{\left\langle\mathbf{r}_{n-1}, P \mathbf{r}_{n-1}\right\rangle} .
$$

We consider the standard choice $\beta=\max \left(\beta^{\mathrm{PR}}, 0\right)$, corresponding to restarting by the CG method when $\beta^{\mathrm{PR}}<0$. For the justification of the CG method for constrained minimization, we refer to $[2,36]$. The $\mathrm{CG}$ algorithm is then summarized in Algorithm 1. Concerning the choice of $\theta_{n}$, we take

$$
\theta_{n}^{\text {opt }}=\frac{-\operatorname{Re}\left\langle\left(\nabla E_{\mathrm{tot}}\right)\left(\phi_{n}\right), \mathbf{p}_{n}\right\rangle\left\|\mathbf{p}_{n}\right\|}{\operatorname{Re}\left[\left(\nabla^{2} E_{\mathrm{tot}}\right)\left(\phi_{n}\right)\left[\mathbf{p}_{n}, \mathbf{p}_{n}\right]-\lambda_{n}\right]},
$$

and use the same step size control as in the steepest descent algorithm. In practice, these precautions for checking the descent direction and using a stepsize control technique are important when located in the neighborhood of a minimum. When a minimum is approximately obtained, $\mathbf{p}_{n}$ is always a descent 


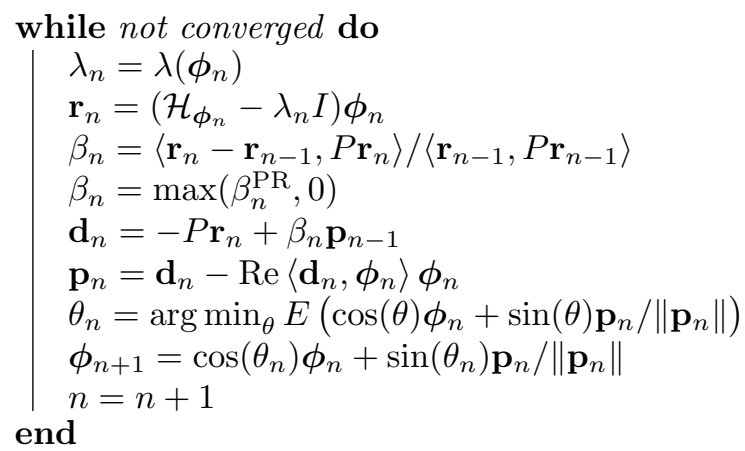

Algorithm 1: The Preconditioned Conjugate Gradient (PCG) method as implemented in BEC2HPC.

direction and the stepsize choice (2.6) decreases the energy functional. Finally, we use the following robust stopping criterion (see [11])

$$
E_{\mathrm{err}}^{n}:=\left|E_{\mathrm{tot}}\left(\phi_{n+1}\right)-E_{\mathrm{tot}}\left(\phi_{n}\right)\right| \leq \varepsilon .
$$

This finally leads to the following PCG algorithm.

Let us now focus on the important question of building an efficient and robust preconditioner $P$ as a modification of the descent direction, leading then to a closer point to the minimum

$$
\mathbf{d}_{n}:=-P\left(\mathcal{H}_{\phi_{n}}-\lambda_{n} I\right) \phi_{n} .
$$

The preconditioner should be an approximation of the inverse of the Hessian matrix of the problem. A first preconditioner [11] uses only the kinetic energy term through an adaptive preconditioner

$$
P_{\Delta}^{n}=\left(\alpha_{\Delta}^{n} I-\Delta / 2\right)^{-1}
$$

where $\alpha_{\Delta}^{n}$ is a positive shifting constant defined by the characteristic energy

$$
\alpha_{\Delta}^{n}=E_{\mathrm{kin}}\left(\phi_{n}\right)+E_{\text {pot }}\left(\phi_{n}\right)+E_{\text {int }}\left(\phi_{n}\right)>0 .
$$

This preconditioner provides a convergence independent of the grid refinement and is diagonal in the Fourier space. Another natural approach is to use the diagonal preconditioner based on the potential and nonlinear interaction terms

$$
P_{V}^{n}=\left(\alpha_{V}^{n} I+V+f\left(\left|\phi_{n}\right|^{2}\right)\right)^{-1},
$$

with $\alpha_{V}^{n}=\alpha_{\Delta}^{n}$. This preconditioner is well-adapted to large nonlinearities and large domains. Finally, to get a stable performance independent of the size of the domain or the spatial resolution, we can define the combined preconditioners

$$
P_{\mathrm{C}_{1}}^{n}=P_{V}^{n} P_{\Delta}^{n}, \quad P_{\mathrm{C}_{2}}^{n}=P_{\Delta}^{n} P_{V}^{n}
$$

or a symmetrized version

$$
P_{\mathrm{C}}^{n}=P_{V}^{n, 1 / 2} P_{\Delta}^{n} P_{V}^{n, 1 / 2} .
$$

Let us now analyze the computational cost of the PCG. The application of the operator $P_{V}$ is almost free (since it only requires a scaling of $\phi$ ), but the naive application of $P_{\Delta}$ requires a FFT/IFFT pair. However, since we apply the preconditioners after and before an application of the Hamiltonian, we can reuse the FFT and IFFT computations, so that the application of $P_{\Delta}$ does not require any additional Fourier transform. Similarly, the use of $P_{\mathrm{C}_{1}}$ and $P_{\mathrm{C}_{2}}$ only needs one additional Fourier transform per iteration, and that of the symmetrized version $P_{\mathrm{C}}$ two.

To summarize, the cost in terms of Fourier transforms per iteration for the rotating GPE model is

- no preconditioner: 3 FFTs/iteration (get the Fourier transform of $\phi$, and two IFFTs to compute $\Delta \phi$ and $L_{z} \phi$ respectively), 
- $P_{\Delta}$ or $P_{V}: 3 \mathrm{FFTs} /$ iteration,

- non-symmetric combined $P_{\mathrm{C}_{1}}$ or $P_{\mathrm{C}_{2}}: 4$ FFTs/iteration,

- symmetric combined $P_{\mathrm{C}}: 5 \mathrm{FFTs} /$ iteration.

Note that this total cost might be different for another type of GPE model e.g. when a nonlocal dipoledipole interaction is included [10,20].

\section{Implementation of the PCG method}

In this section, we present an implementation of the previous PCG method for computing the stationary states of the GPE. The resulting code, called BEC2HPC, is developed in C++ and uses MPI communications for distributed computing. The code is available online at https://team.inria.fr/bec $2 \mathrm{hpc} /$. A Python interface is provided for defining the physics of the problem and external visualization tools such as Paraview can be used to exploit the results of the simulations. The code takes advantage of existing HPC libraries and even if we focus here on the implementation of the PCG methods, it can itself be leveraged to implement other spectral methods or solve e.g. problems related to the dynamics of BECs. In particular, schemes for simulating the dynamics of the GPE will be included in a future version of BEC2HPC.

\subsection{Implementation of FFT-based schemes in distributed memory}

The wave function $\phi$ is truncated to a square domain with periodic boundary conditions and discretized on a grid with $n_{x}, n_{y}, n_{z}$ points along each dimension $x, y$ and $z$, respectively. This grid structure leads to multidimensional arrays on which we perform one-dimensional discrete Fourier transforms along each dimension independently. For a 2D domain, we compute the transform of each column and each row of a matrix to obtain $\left[\left[\partial_{x}\right]\right]$ and $\left[\left[\partial_{y}\right]\right]$. In distributed-memory, these arrays are divided among a set of processes which each runs in their own memory address space. Several librairies implement parallel FFT algorithms working on distributed data. These codes use either 1D slab (FFTW [39]) or 2D pencil decompositions (PFFT [54], P3DFFT [53], 2DECOMP\&FFT [46]). The idea is to simply reuse serial FFT algorithms in local memory. A 1D domain distribution along the dimension $X$ (Figure 1) of a 3D domain allows to compute both $\left[\left[\partial_{y}\right]\right]$ and $\left[\left[\partial_{z}\right]\right]$ without any interprocess communications as the grid elements involved in the computation of each 1D FFT along the $Y$ and $Z$ lines are assigned to the same process. A permutation of the axis of the array makes the computation along $X$ local at the cost of all-to-all communication to transpose the distributed array. A 2D pencil domain decomposition only maintains the data placement useful for one direction and requires transpositions for each of the other directions but it exhibits more parallelism as a 1D decomposition along the dimension $X$ is limited to using at most $n_{x}$ processes.

BEC2HPC is based on FFTW 3.3 (see [39]). We use the 1D block distribution of the data (distributed along the first dimension), the sequential FFT routine and the distributed transpose routine provided by the library. Using the FFTW's advanced interface, one can perform multiple sequential 1D complex FFT simultaneously on non-contiguous data (fftw_plan_many_dft), allowing to compute directly the FFTs along $Y$ and $Z$ on each process. The MPI transpose routine used internally by FFTW for multidimensional transforms is also exposed in its API ( $f f_{t w}$ mpi_plan_many_transpose for complex numbers) and is called directly for the computation of $\left[\left[\partial_{x}\right]\right]$. FFTW comes with a set of routines for the creation of the domain distribution (dividing the data among the MPI processes), the allocation of memory (with due consideration to alignment for SIMD instructions and the extra memory that might be needed for a data redistribution). Computation of a Fourier transform is preceded by a preprocessing phase selecting at runtime an efficient strategy for computing a transform on the current hardware: it creates a plan (an opaque data type) describing the algorithm and transforms can then be executed repeatedly.

\subsection{BEC2HPC parallel code design}

Building FFT-based schemes on the foundation of FFTW is a good starting point as this library is fast, freely available and became defacto standard for scientific softwares or for benchmarking other FFT librairies. In addition, other FFT librairies such as the Intel Math Kernel Library (MKL) offers FFTW interfaces without changing the program source code. NVIDIA also provides FFTW interfaces to the cuFFT library. However, it seems important to integrate in the initial design more general data 


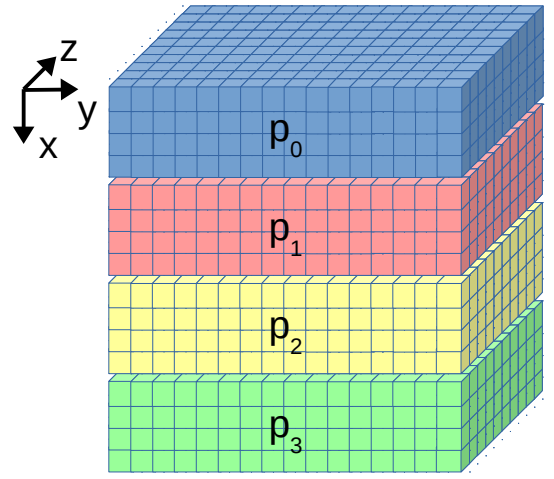

(a) A 1D domain decomposition in the $X$ direction.

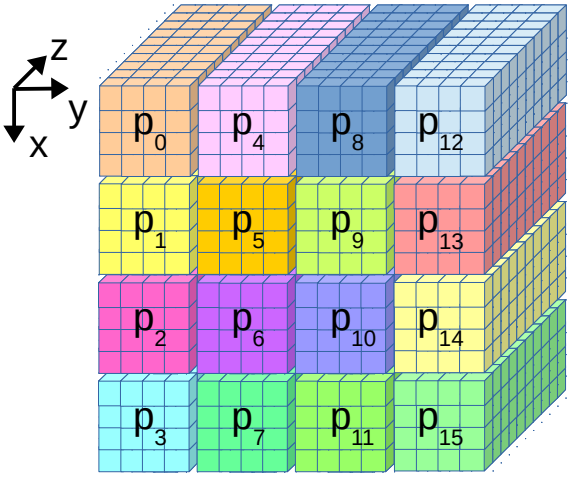

(b) A 2D (pencil) domain decomposition.

Figure 1: Decomposition strategies for a 3D domain.

distributions as well as the possibilities of using hybrid MPI-plus-thread approach in the future. On multicore clusters, OpenMP directives can be used to distribute the set of serial 1D FFT to be performed within a MPI process. Multi-threaded FFT algorithms can also replace serial FFT to leverage parallelism along a second dimension as FFTW provides multi-threaded transforms with exactly the same API as the serial version.

Currently, BEC2HPC only uses FFTW but we avoided binding the code directly to this third-party library. FFTW's routine calls and data types are encapsulated and BEC2HPC defines its own interface for data distribution, transforms and transposition. In BEC2HPC, the data distribution is described by a Map object taking up ideas of well established parallel scientific libraries [37]. Map objects contain the details of the block distribution along each dimension (including domain dimensions, local and global partitioning indices) and distributed objects are subsequently created from Map objects. The distributed arrays class gives methods for creating multidimensional arrays, accessing local array elements. It also provides foreach loops for performing pointwise operations for each domain element and parallel reduce using $\mathrm{C}++11$ lambda. It hides much of the complexity of the data distribution and facilitates the application of operators and the computation of norms and convergence criteria. As a result, the grid distribution, the local indices and MPI routine do not appear on the implementation of the PCG algorithm, improving the code readability. These classes also provide a high-level parallel abstraction layer that can evolve to support different memory layout and programming paradigm for multicore processors.

The spectral numerical method itself is implemented on top of this using a modular design to ease the change of components such as preconditioners or stopping criterias as described in Section 2. At each iteration, transforms are never computed twice and operators such as $[[\Delta]]$ or $\left[\left[L_{z}\right]\right]$ are stored along side $\phi_{n}$ to be used on each components.

\section{Getting started with BEC2HPC}

This section proposes a first BEC2HPC example and shows off the basics usage of BEC2HPC. BEC2HPC provides both a $\mathrm{C}++$ and python interfaces. In this paper, we present the Python interface built using the Boost Python Library, a framework for exposing the $\mathrm{C}++$ classes functions and objects to Python.

\subsection{Installation}

The build process of BEC2HPC is managed by CMake and requires a $\mathrm{C}++11$ compiler, a MPI library and several external libraries compiled with parallel support (namely Boost, FFTW and HDF5). Python3 is also needed for running most of the examples. These softwares are usually pre-installed on HPC machines. We also provide a Vagrant setup to automatically configure a virtual computing environment suitable for compiling and running BEC2HPC on personal computers. Vagrant [41] is a popular tool for building virtual machines (or containers) from a configuration file describing the machine setup and the necessary steps to create a ready-to-use machine. The virtual machine behaves like a separate computer system and can be accessed via a SSH connection as if it was a remote physical machine. Figure 2 shows how to create a Vagrant managed virtual machine for BEC2HPC. Within the 


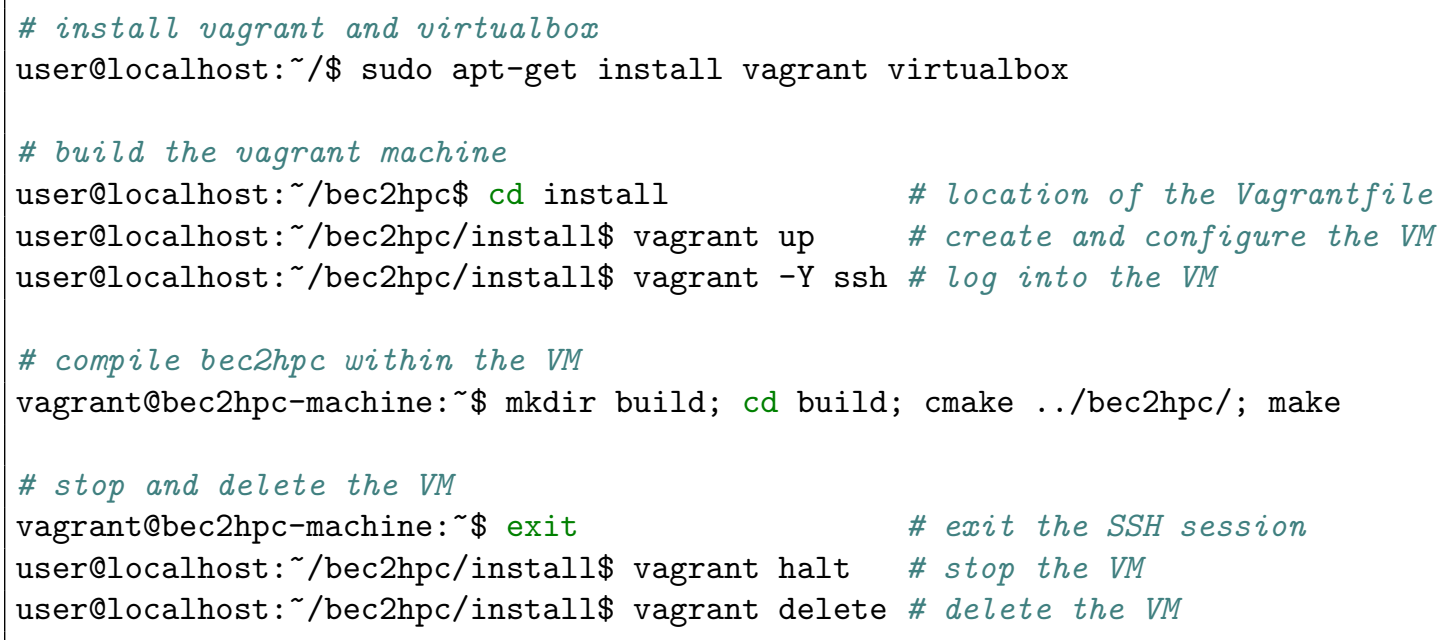

Figure 2: Creating a Vagrant managed Virtual Machine (VM) for BEC2HPC.

virtual machine, the bec2hpc directory is shared with the host and therefore contains the source code. build is an out-of-source build directory to keep separate the files generated by the compilation.

\subsection{A first example}

Let us consider the physical problem governed by the following GPE

$$
i \partial_{t} \psi(\mathbf{x}, t)=\left(-\frac{1}{2} \Delta+V(\mathbf{x})+\beta|\psi(\mathbf{x}, t)|^{2}-\mathbf{\Omega} \cdot \mathbf{L}\right) \psi(\mathbf{x}, t),(\mathbf{x}, t) \in \mathbb{R}^{d} \times \mathbb{R}^{*+},
$$

where $V(\mathbf{x})$ is the external confining potential, $\beta$ is the nonlinearity strength describing the interaction between atoms of the condensate, $\boldsymbol{\Omega}$ is the angular velocity vector and $\mathbf{L}$ is the angular momentum operator. By default in BEC2HPC, the rotation term is such that $\boldsymbol{\Omega} \cdot \mathbf{L}=\omega L_{z}=-i \omega\left(x \partial_{y}-y \partial_{x}\right)$ (i.e. $\left.\boldsymbol{\Omega}=(0,0, \omega)^{t}\right)$, and the nonlinearity is cubic, i.e. $f(|\psi|)=\beta|\psi|^{2}$. The harmonic potential $V$ is given by

$$
V(x, y, z)=\frac{1}{2}\left(\gamma_{x}^{2} x^{2}+\gamma_{y}^{2} y^{2}+\gamma_{z}^{2} z^{2}\right)
$$

with $\gamma_{x}=\gamma_{y}=\gamma_{z}=1$ per default in $3 \mathrm{D}$, and $\gamma_{x}=\gamma_{y}=1, \gamma_{z}=0$ in $2 \mathrm{D}$. The predefined initial guess is the Thomas-Fermi approximation

$$
\phi_{0}=\frac{\phi_{\beta}^{\mathrm{TG}}}{\left\|\phi_{\beta}^{\mathrm{TG}}\right\|_{2}}, \text { with } \phi_{\beta}^{\mathrm{TG}}= \begin{cases}\sqrt{\frac{\mu_{\beta}^{\mathrm{TG}}-V(\mathbf{x})}{\beta}}, & \text { if } \mu_{\beta}^{\mathrm{TG}}>V(\mathbf{x}), \\ 0, & \text { otherwise }\end{cases}
$$

where the eigenvalue approximation $\mu_{\beta}^{\mathrm{TG}}$ is given by

$$
\mu_{\beta}^{\mathrm{TG}}=\frac{1}{2} \begin{cases}\left(4 \beta \gamma_{x} \gamma_{y} / \pi\right)^{1 / 2}, & d=2, \\ \left(\frac{15}{4 \pi} \beta \gamma_{x} \gamma_{y} \gamma_{z}\right)^{2 / 5}, & d=3 .\end{cases}
$$

The stopping criterion is fixed by default to $\mathcal{E}_{\text {err }}^{n}:=\left|E_{\text {tot }}\left(\phi_{n+1}\right)-E_{\text {tot }}\left(\phi_{n}\right)\right| \leq \varepsilon$, with $\varepsilon=10^{-12}$.

We consider now that we want to compute the ground state of a $2 \mathrm{D}$ rotating condensate with a cubic nonlinearity and a harmonic potential (setting $\gamma_{x}=\gamma_{y}=1, \gamma_{z}=0$ ). Figure 3 shows a first example of the BEC2HPC API. The computational domain and spatial mesh sizes are chosen respectively as $[-16,16]^{2}$ and $h=\frac{1}{4}(M=128)$. This example can be run within the virtual machine with mpiexec -n 1 python simple-example.py. The physics and solver parameters are listed in two distinct python hashmaps. The physics submodule can be used to generate the initial guess. The 


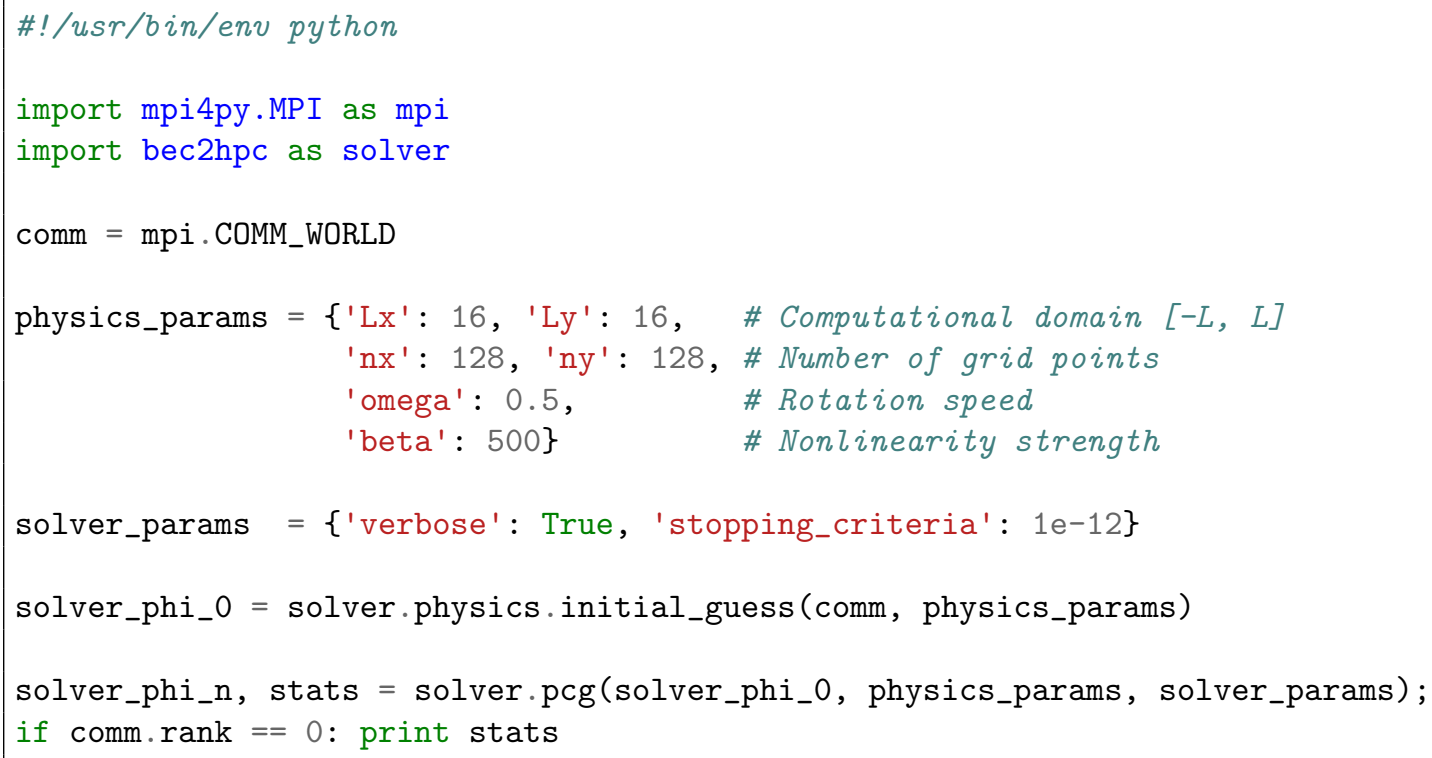

Figure 3: A first example.

pcg method returns the solution solver_phi_n along with information about the solver execution in a hashmap stats. This includes in particular the energy at the final state, the convergence history and the computational time to reach the stationary state (Figure 4). The solution solver_phi_n can be saved in a HDF5 file as shown in Figure 5. HDF5 [40] is a widely-used standard binary format for storing numerical data and BEC2HPC uses the Parallel HDF5 library to efficiently write on disk in a parallel environment (using MPI-I/O). The solution can later be postprocessed or visualized with a variety of tools such as Python or Paraview (see also Section 5.3). The solution can also be transferred to a single processor by using local_phi = solver array.gather(phi). For instance, it can be used to plot phi_n without saving it to a file. Figure 6 shows how to load a HDF5 file and plot the density function $\rho=|\phi|^{2}$ in Python. The provided function bec2hpc.utils.plot2d uses matplotlib internally. Figure 7 shows the square of the amplitude of the wave function on the computational domain for $\omega=0.5$ and $\beta=500$. The stats output of solver.pcg can be saved as a JSON file (or in any human-readable format) along side the input parameters to retain the information of a simulation run.

\section{BEC2HPC advanced usage}

After this step-by-step example on a model problem, we now describe in more details some of the code functionalities for defining the physical problem and the numerical scheme. We also present along the way how to manage the distributed data structures efficiently in Python.

\subsection{Defining the initial data}

The iterative method for computing the solution of the minimization problem needs to be initialized with a guess. As the minimization algorithm is a local optimization procedure, the choice of the initial guess can lead to a local minimum and therefore a different final converged state [11]. The initial guess is usually an approximation of the solution of a simpler problem and BEC2HPC provides initial data typically found in the literature such as a centered Gaussian for fast rotations or the Thomas-Fermi approximations (4.16) for strong nonlinear interactions [10, 11, 61]. In 2D, the centered Gaussian is defined by

$$
\phi(\mathbf{x})=\frac{(1-\omega) \phi_{a}(\mathbf{x})+\omega \phi_{b}(\mathbf{x})}{\left\|(1-\omega) \phi_{a}(\mathbf{x})+\omega \phi_{b}(\mathbf{x})\right\|},
$$




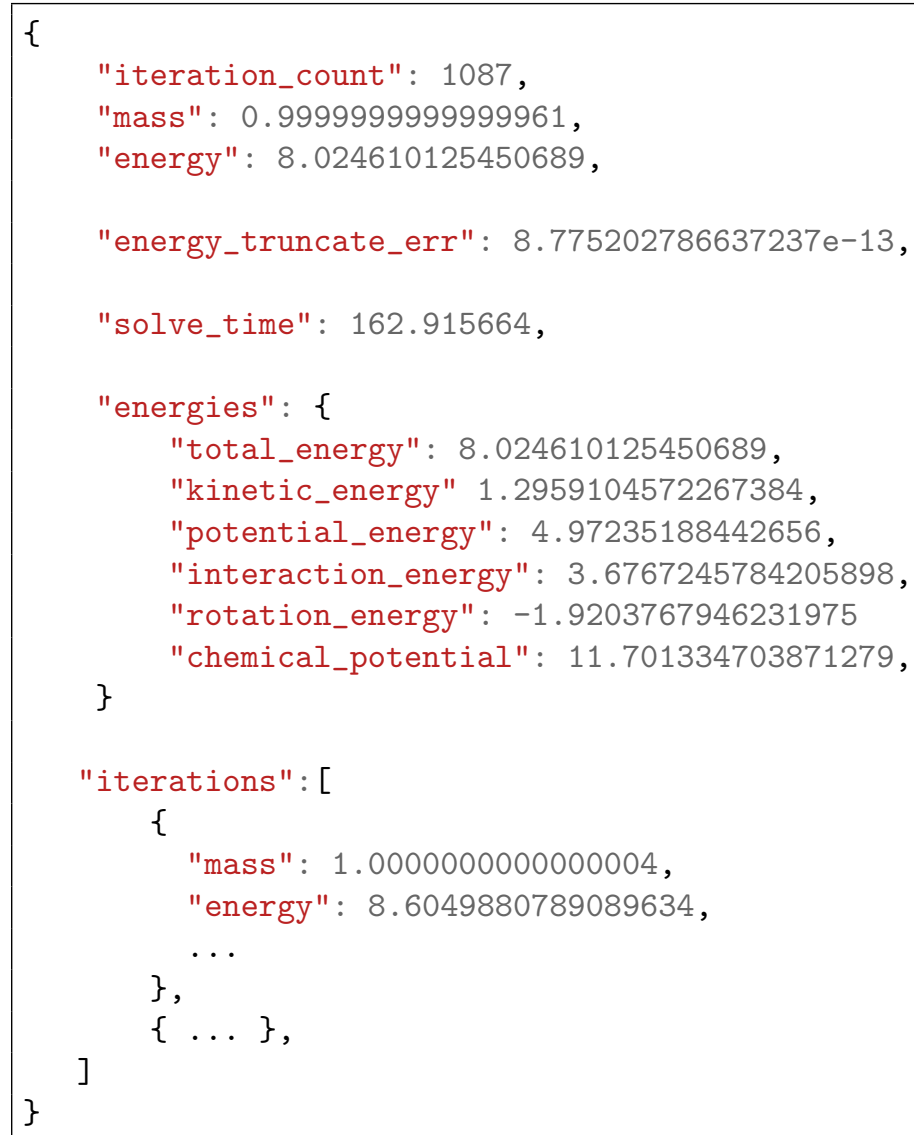

Figure 4: Output of the solver.pcg function in a JSON format

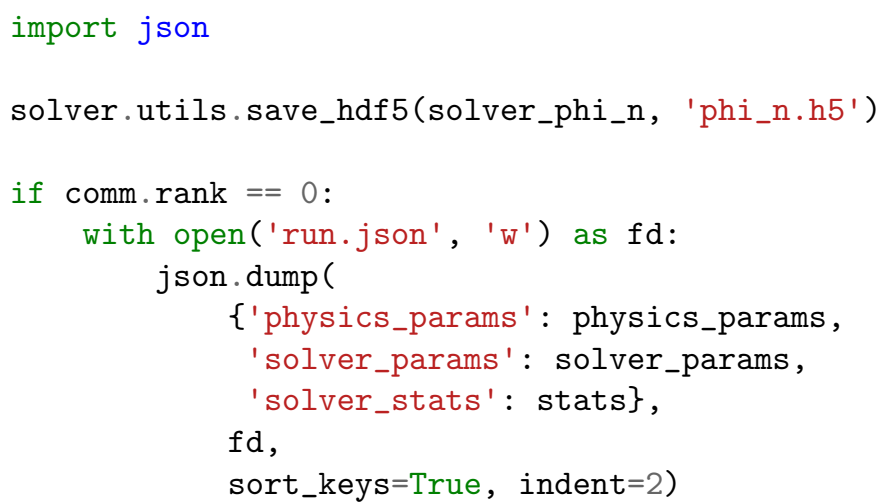

Figure 5: Saving the ouput of the solver.pcg function. 


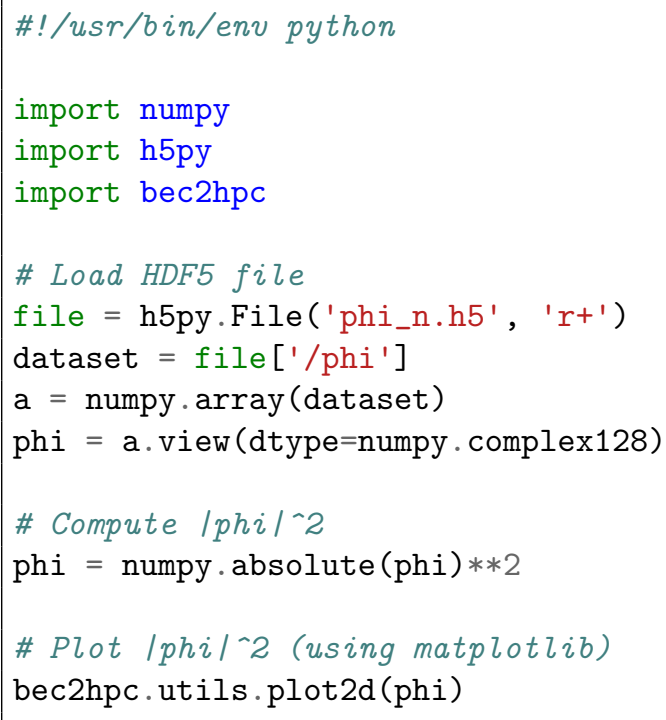

Figure 6: Compute and plot the density function $|\phi|^{2}$.

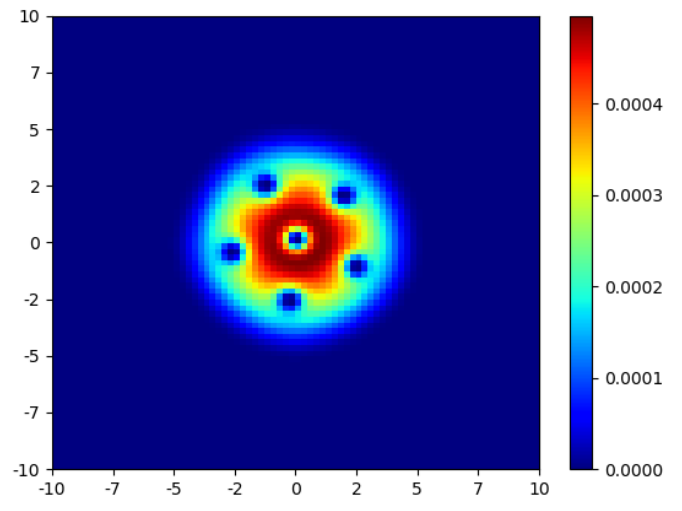

Figure 7: Density function $|\phi|^{2}$ of the ground state. 


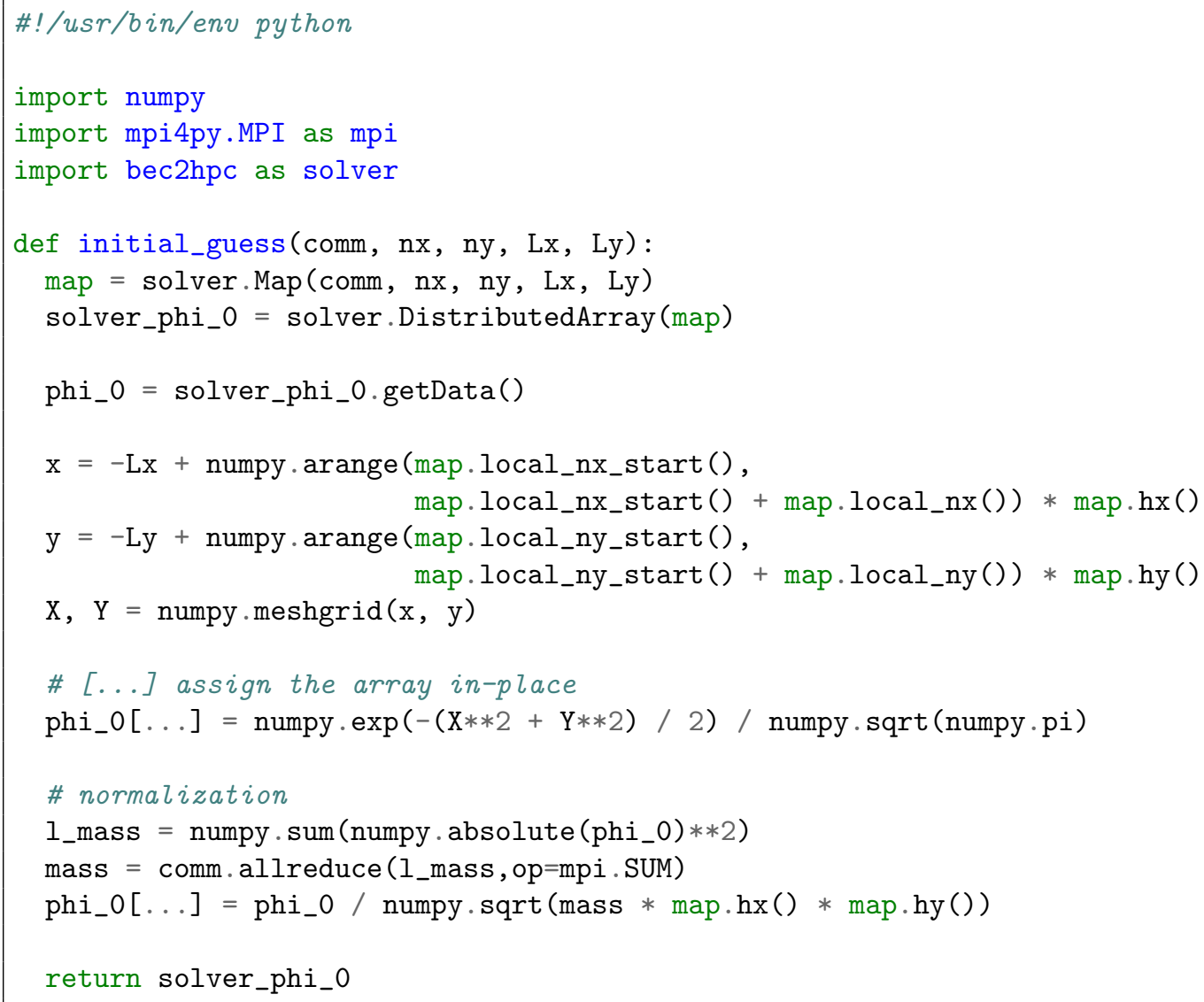

Figure 8: Defining a initial guess.

where

$$
\phi_{a}(\mathbf{x})=\frac{1}{\sqrt{\pi}} e^{-\left(\gamma_{x} x^{2}+\gamma_{y} y^{2}\right) / 2}, \quad \phi_{b}(x)=\left(\gamma_{x} x+i \gamma_{y} y\right) \phi_{a}(\mathbf{x})
$$

It is also possible to provide your own initial guess to the pcg method once you become familiar with the BEC2HPC distributed array class. Figure 8 shows how to implement the gaussian defined by $(5.17)$ for $\gamma_{x}=\gamma_{y}=1$ and $\omega=0$, a simple choice for weak nonlinear interaction and subcritical frequencies.

In Python, the elements of a DistributedArray are accessible as a numpy array by using the getData() method. This method returns a numpy array containing the local part of the distributed array without making a copy of the underlying data. The numpy array is actually a view of the original data and modifying the numpy array changes the distributed array (and vice versa). The data distribution of an array is described by a Map object and can be retrieved using the getMap() method on the array. The python ellipsis syntax ('...') is used to assign the result of the calculation to the original numpy array memory buffer (otherwise, with a simple array assignment, phi_0 would not be referencing the BEC2HPC array anymore).

\subsection{Defining the trapping potential}

BEC2HPC comes with predefined potential functions for an harmonic trap (4.15) and other potentials with added potential terms such as the harmonic-plus-quartic potential

$$
V(\mathbf{x})=(1-\alpha) \sum_{\nu=x, y} \gamma_{\nu} \nu^{2}+\frac{\kappa\left(x^{2}+y^{2}\right)^{2}}{4} \begin{cases}+0, & d=2 \\ +\gamma_{z}^{2} z^{2}, & d=3\end{cases}
$$

Figure 9 shows how to provide a user-defined potential in Python. Either Python function or a lambda form could be specified on the parameter list describing the physics of the problem. One can also 


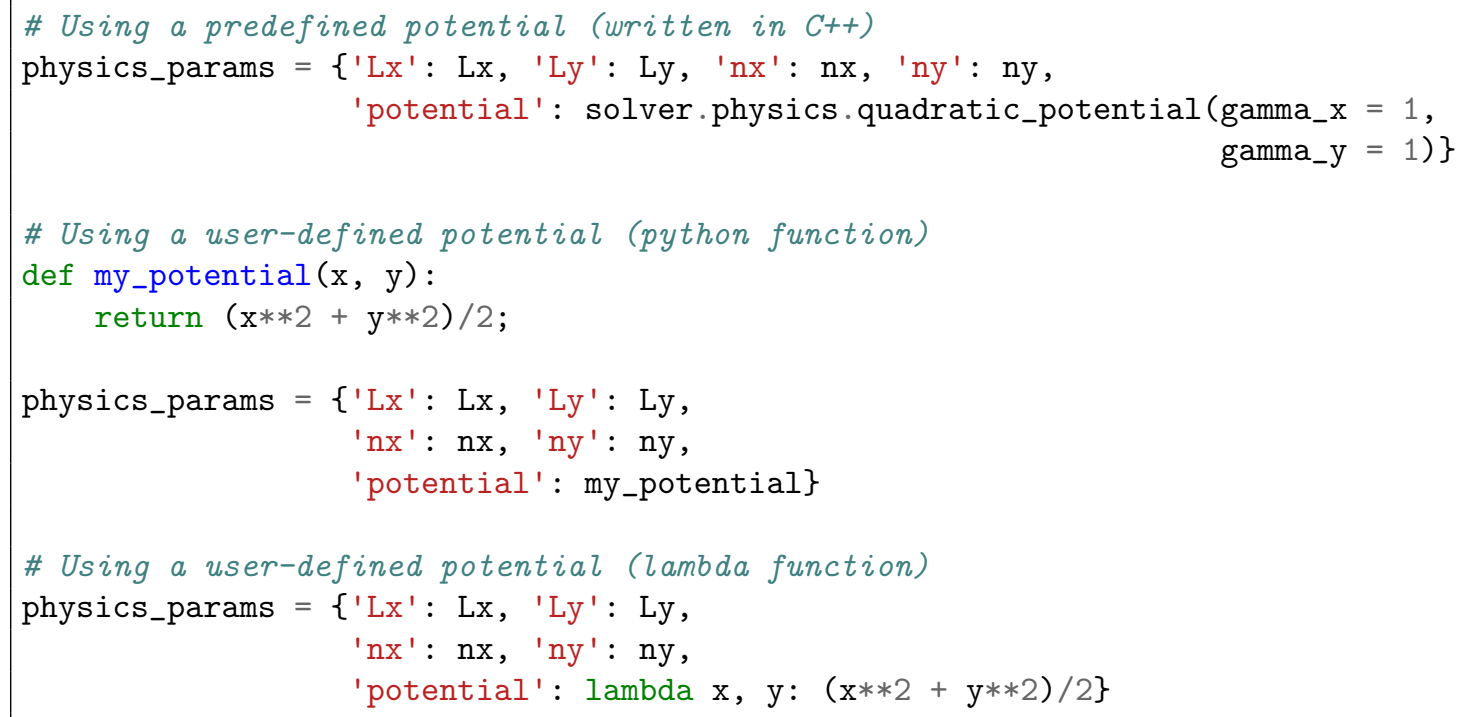

Figure 9: User-specified potentials.

implement its own $\mathrm{C}++$ potential class by deriving the $\mathrm{C}++$ Potential abstract class and instantiating it in Python. On the same model, it is possible to redefine the nonlinearity part of the equation as well as the stopping criteria and the preconditioner to be used during the nonlinear conjugate gradient.

\subsection{D simulation and visualization}

The previous examples were in 2D for conciseness but BEC2HPC has been designed with large 3D problems in mind. For code maintenance purpose, 2D and 3D cases share the same code base internally (there is no code duplication). The functions described in $2 \mathrm{D}$ in this paper are all available in 3D as well. Running a 3D simulation only requires to define the computational boundary and the number of grid points in the third dimension (by adding Lz and $\mathrm{nz}$ in the physics parameter list).

$3 \mathrm{D}$ visualizations of large problems might be more tricky but thankfully, specialized applications such as ParaView can be leveraged [4]. ParaView cannot read directly a HDF5 file as it needs information concerning the semantic of the data. Such information can be provided by a simple XDMF file describing the data scheme. XDMF (eXtensible Data Model and Format) uses XML to store metadata and refers to external HDF5 files for the values themselves. It is a standard way format to describe the raw data produced by HPC codes and BEC2HPC provides a function bec2hpc.utils.save_xdmf (phi_n, 'phi_n.xdmf ') to create a XDMF describing the semantic of a BEC2HPC HDF5 output created with the call bec2hpc.utils.save_hdf5(phi_n, 'phi_n.hdf5).

The ParaView Python API gives full access to its data analysis and visualization capabilities. It can be used to programmatically visualize the result of a BEC2HPC simulation. When running multiple tests, it might be handy to define a visualization with the ParaView application, save the visualization state and then reload it from Python. An example to load a ParaView state file (a file with a .pvsm extension) is available in the examples/paraview directory of BEC2HPC.

\subsection{Grid manipulation}

In order to both limit the number of iterations and reach the lowest energy state, the initial guess of a simulation must be chosen close to the expected solution. Several choices for an initial guess are described in subsection 5.1. Another approach to define an initial guess is to use the results of a previous simulation. It can give good results when the simulation parameters varies only slightly or when we make them gradually changing. For large grid sizes, one can use the results of the same simulation performed on coarser grid involving less points. It is also possible to tune the size of the domains once we learned on a fastest, less accurate simulation the space occupied by the condensate for a given rotational speed and potential.

The mapping of a coarse grid data on a finer grid is done using an interpolation. Figure 10 gives an example for linearly interpolating a 2D grid in Python. In this example, the coarse grid solution is simply 
gathered on a single processor and the interpolation is done in sequential using the interpolate.interpn function of the SciPy library. This process can be repeated on several grid levels to form the hierarchical multigrid strategy presented in $[11,61]$ and implemented with BEC2HPC in Figure 11. The ground state computation begins on a coarse grid of $2^{N_{\text {coarse }}} \times 2^{N_{\text {coarse }}}$ points and the grid is successively refined until a finest grid with $2^{N_{\text {fine }}} \times 2^{N_{\text {fine }}}$. The stopping criteria can be relaxed for the coarse grids as shown in the example.

Nevertheless, strategies based on simple continuation or multigrid strategies should be used carefully since they can also sometimes provide intermediate or final solutions which are not correct. More advanced algorithms $[60,43,59]$ should probably be considered to obtain fast and robust methods.

\section{Numerical examples}

\subsection{Experimental setup}

In this section, we present some results obtained from simulations with BEC2HPC in 2D/3D for fast rotating BECs on a parallel cluster. Experiments were conducted on NIC4, a massively parallel cluster of the University of Liège, installed in the framework of the Belgium consortium of HPC centers (CÉCI) and funded by F.R.S.-FNRS under Grant No. 2.5020.11. It features nodes with two 8-cores Intel E5-2650 processors at $2.0 \mathrm{GHz}$ and $64 \mathrm{~GB}$ of RAM (4 GB/core), interconnected with a QDR Infiniband network.

In the following examples, we consider the cubic nonlinearity and the harmonic-plus-quartic potential (5.19), with $\gamma_{x}=\gamma_{y}=1, \alpha=1.2$ and $\kappa=0.3$. In 3D, we fix $\gamma_{z}=3$. This potential leads to the existence of stationary states for highly rotating BECs for values $\omega$ larger than one, unlike the standard harmonic potential case. We initialize the PCG method with the Thomas-Fermi ansatz (4.16) presented in Section 4.2. We only report the results obtained with the symmetrical version of the combined preconditioner (2.13) as it outperforms the other tested preconditioners. For the stopping criterion, we use $\mathcal{E}_{\mathrm{err}}^{n}:=\left|E\left(\phi_{n+1}\right)-E\left(\phi_{n}\right)\right| \leq \varepsilon$ which is well suited for rotating BECs (to include the non-uniqueness of the minimum up to a rotation).

The experiments presented here were carried out for a wide range of rotation speeds and nonlinearity strengths. The strong scaling of the code is also tested by running the same experiments with an increasing number of MPI processes. To facilitate the testing when several parameters need to vary and to run the solver several times, we provide an easy way to generate and launch a set of experiments. These scripts are included in the example directory of the BEC2HPC distribution. Figure 12 shows how to generate input parameters lists describing the physics for each combination of $\omega$ and $\beta$ where $\omega \in\{1, \ldots, 4.5\}$ and $\beta \in\{1000,5000,10000\}$. The Python dictionary physics_params_set includes a description of the parameters range for $\omega$ and $\beta$. utils.extend_params generates all possible combinations of the given input parameters for the pcg function. In this example, each parameters list is saved in its own JSON file and the utility function gen_id generates a string to name the experiments (for example, 2D__L_20__n_640__Omega_1__Beta_1000.json). These JSON files can then be used to run BEC2HPC as shown in Figure 13. This last script takes a list of JSON files as input parameter and can be used within a submission script for a job scheduler on a HPC machine.

\subsection{Numerical results in $2 D$}

In $2 \mathrm{D}$, the computational domain and mesh sizes are chosen respectively as $[-20,20]^{2}$ and $h=\frac{1}{16}$ $(M=640)$. Concerning the stopping criterion, the tolerance is set to $\varepsilon=10^{-14}$. Figure 14 shows the converged stationary states computed by BEC2HPC for $\beta=1000$ and different rotation speeds. By increasing the rotation velocity, the Bose-Einstein condensate expands into a ring shaped BEC with an increasing central radius. In particular, for $\omega=5$, we get a thin ring with one layer of uniformly spaced vortices. Figure 15 shows additional results for a larger nonlinearity strength, i.e. $\beta=10000$, showing that more vortices characterize the BEC for larger values of $\beta$.

In Figure 16, the number of MPI processes is fixed to $n=32$. We compare the CPU times (in seconds) needed to have the PCG method converging, for various values of $\omega$ and $\beta$. The CPU time is directly related to the number of iterations \#it. In particular, higher rotation speeds $\omega$ and stronger nonlinearities $\beta$ usually require more iterations (and so more simulation times) but it is not always the case. Using a stopping criteria based on the energy difference is advantageous to avoid situations where the residual evolves without changing the energy.

Figure 17 presents the number of iterations \#it and the CPU time required to compute the ground state for $\beta=1000$ and different numbers of MPI processes. This shows how the computational time 


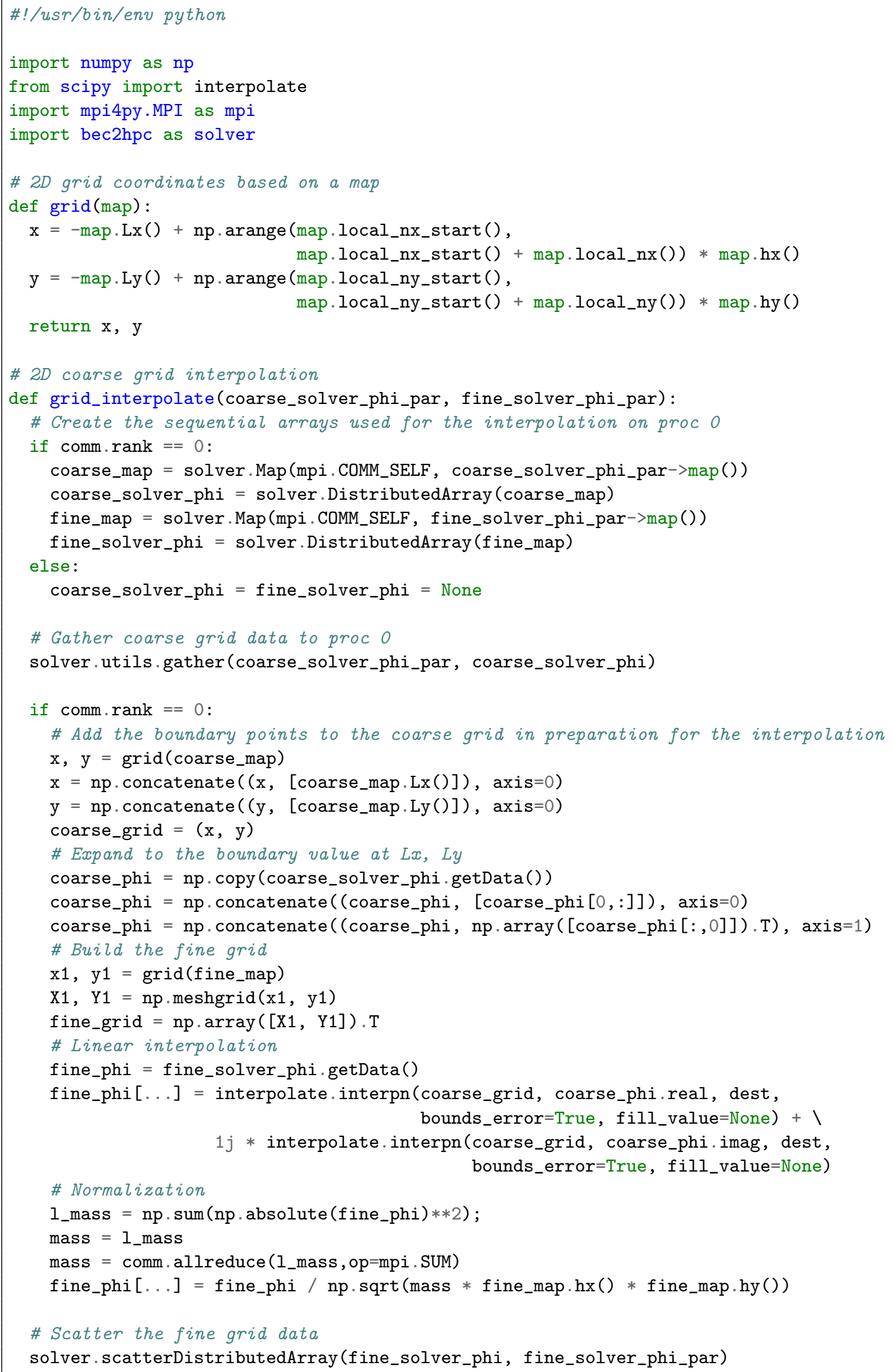

Figure 10: Coarse grid interpolation. 


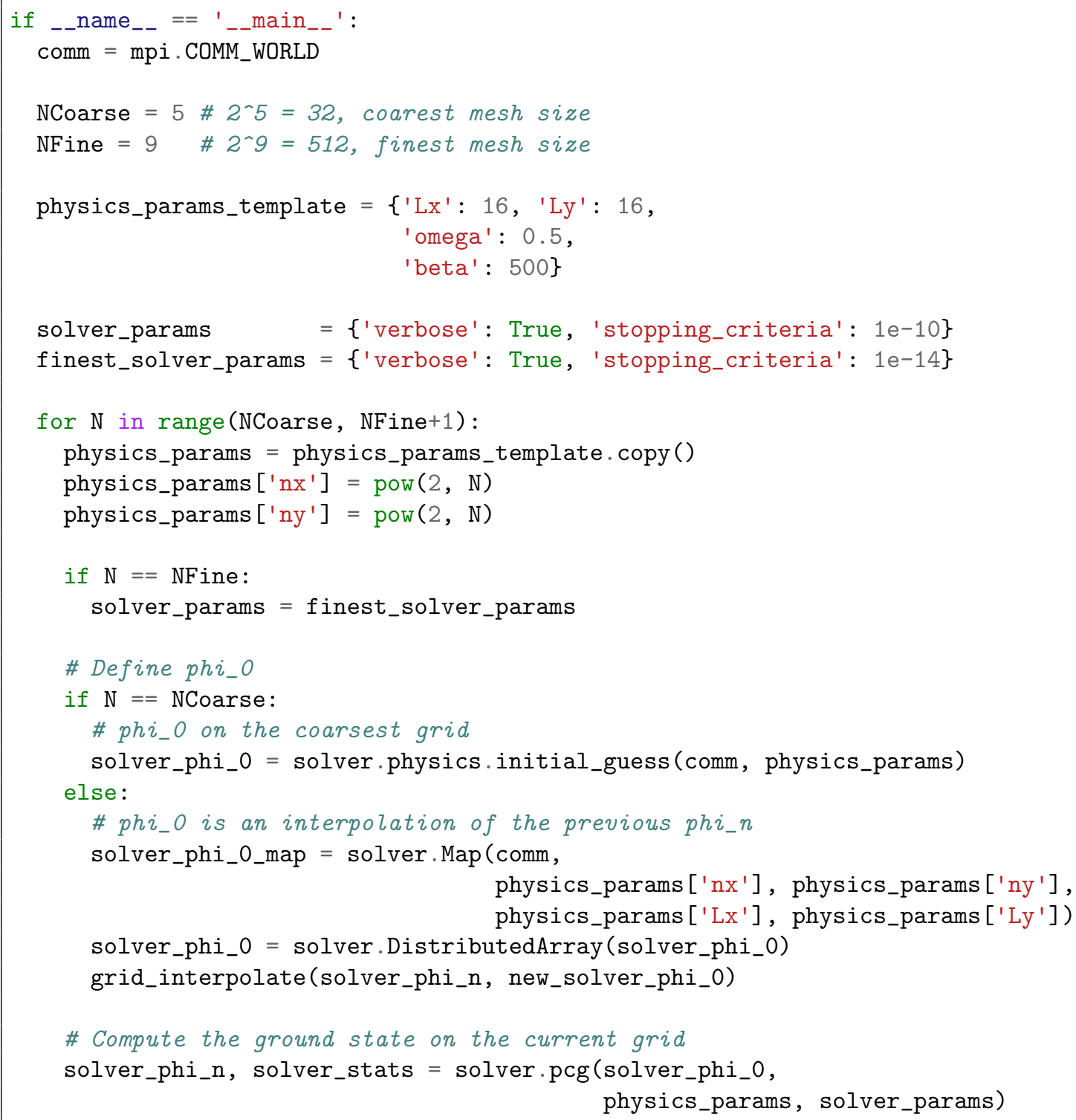

Figure 11: A multigrid approach for defining the initial guess. 


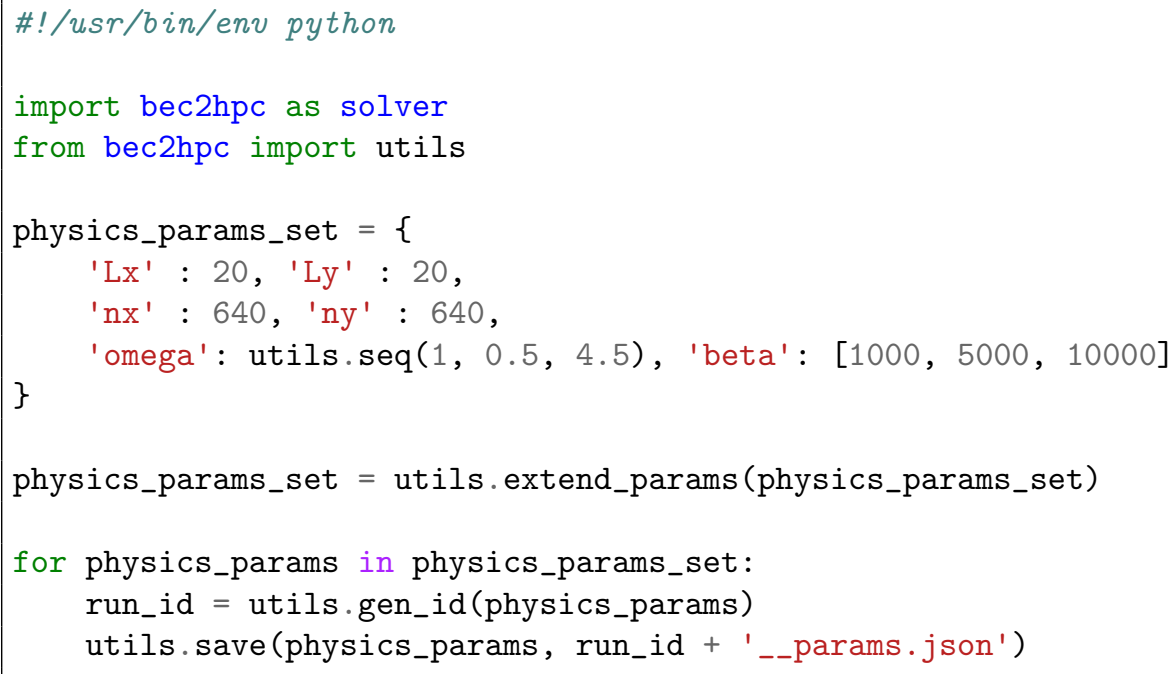

Figure 12: Generating a JSON file for each combination of the parameters $\omega$ and $\beta$.

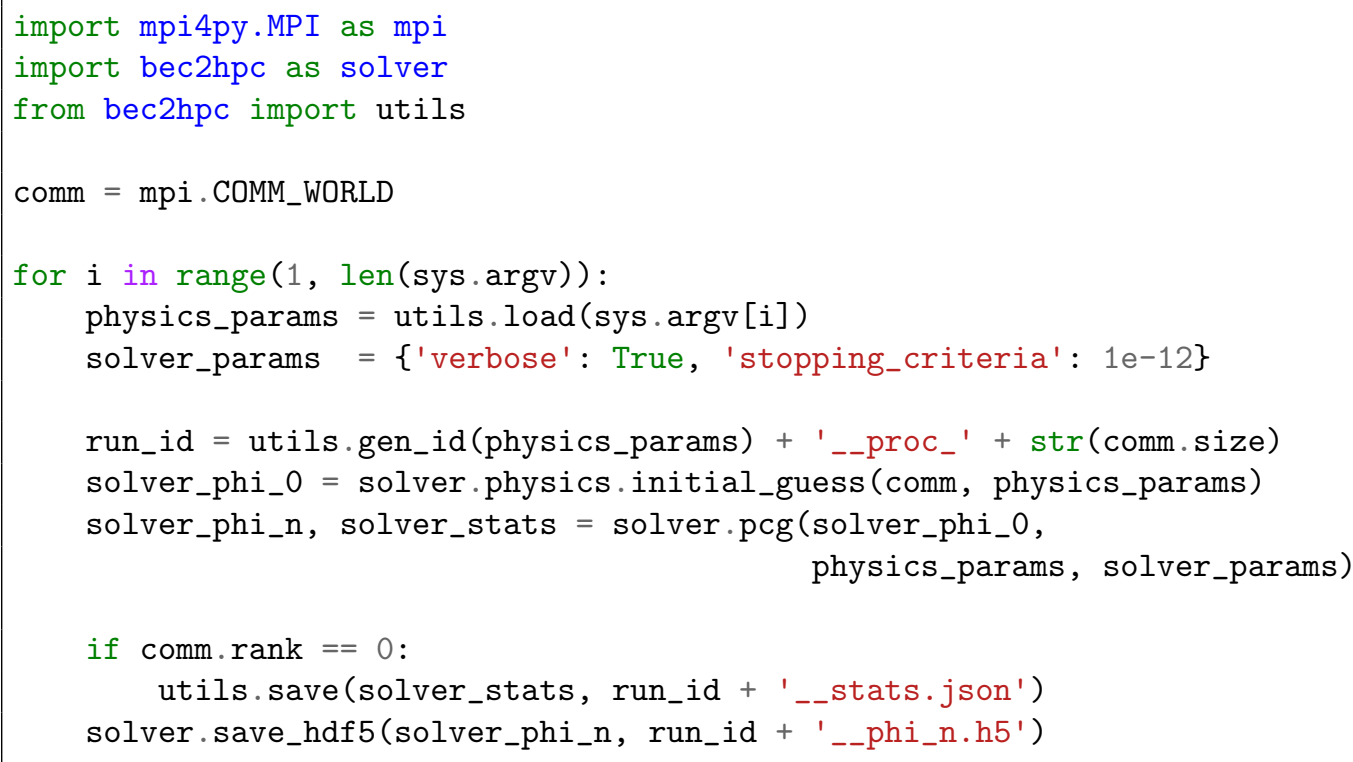

Figure 13: Running BEC2HPC using JSON files as the description of the physical setups. 


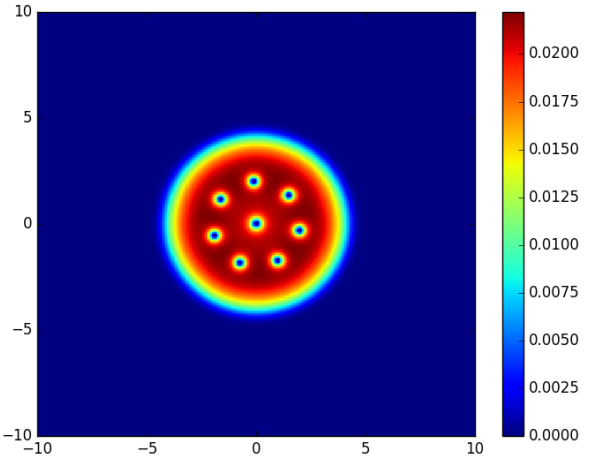

(a) $\beta=1000, \omega=1$

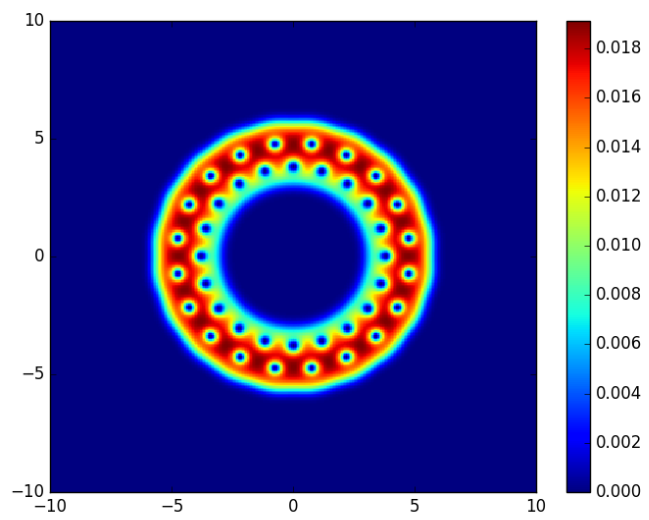

(c) $\beta=1000, \omega=2.5$

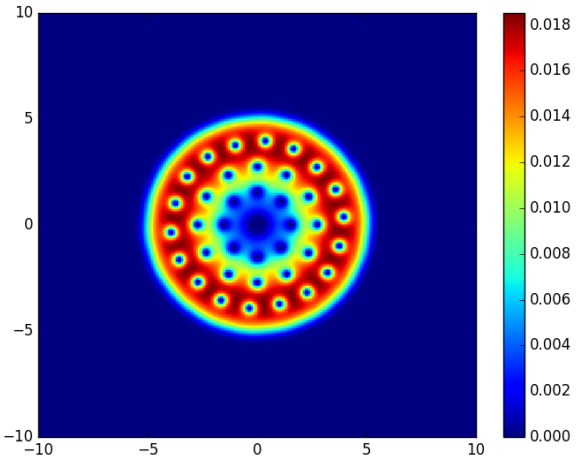

(b) $\beta=1000, \omega=2$

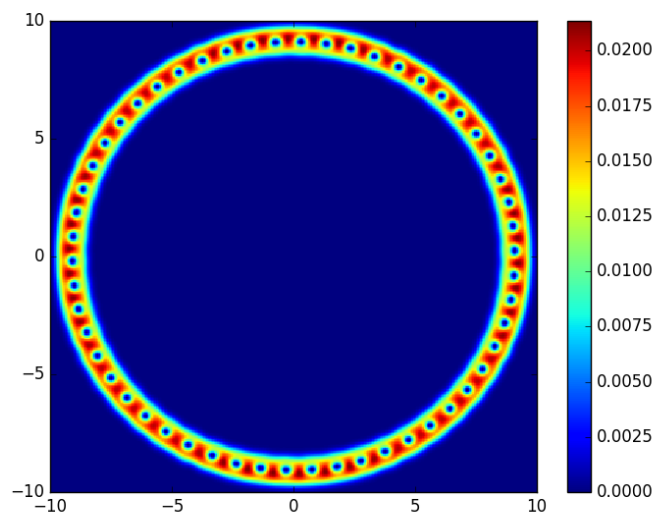

(d) $\beta=1000, \omega=5$

Figure 14: Contour plots of the density function $\left|\phi_{g}\right|^{2}$, for $\beta=1000$ (procs $=32$ ).

scales with the number of MPI processes. Running the computation on 32 cores is 15-20 times faster than on a single core. The scalability results are consistent with the scalability of the 2D FFT algorithm in distributed memory. The number of iterations varies slightly with the number of processors but the same final state is nonetheless reached independently of the number of MPI processes.

Finally, Figure 18 shows the resolution of a very difficult $2 \mathrm{D}$ problem $(\beta=10000, \omega=5)$ on a finer grid $(M=2048)$ using 32 and 128 processors. A fast and precise resolution of such challenging problems is only possible thanks to the robustness of the numerical method and its parallelization implementation.

\subsection{Numerical results in $3 D$}

We solve now various $3 \mathrm{D}$ problems. The computational domain is $[-8,8]^{3}$ and the tolerance of the stopping criterion is set to $\varepsilon=10^{-12}$. Figure 19 presents the results of four experiments for the mesh size $h=\frac{1}{8}(M=128)$. 3D simulations allow to visualize the torus shape of the BEC as well as the vortices lines. The scalability results presented in Table 20 are again consistent with the scalability of the 3D FFT algorithm and the cost of parallel transpose algorithms. Running on 32 cores can be more than 5 times faster than on 4 cores but the speedup is reduced when the number of iterations increases unfavorably. According to our experiments, this instability in the number of iterations occurs when the grid resolution is not fine enough. Figure 21 presents the results of the same experiments on a finer grid with $h=\frac{1}{16}(M=256)$ for 32 and up to 256 cores. By refining the grids, each iteration is more costly in computational time and the resolution also takes more iterations. The computations become rapidly expensive. For example, on this finer grid, more than 3 hours are needed to solve the $\beta=10000, \omega=3$ test case on 256 processors. 


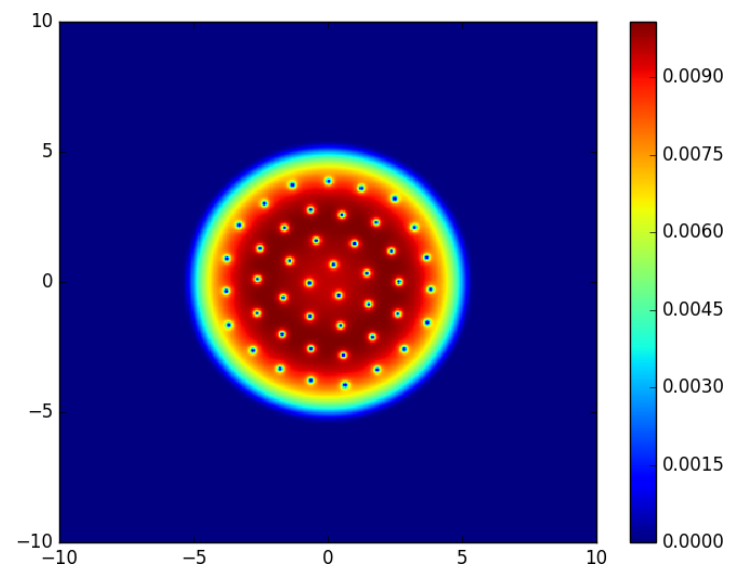

(a) $\beta=10000, \omega=1.5$

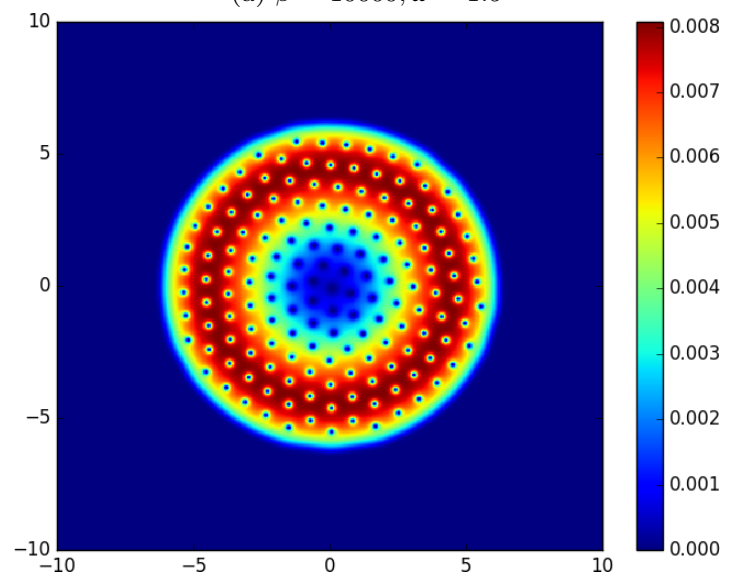

(c) $\beta=10000, \omega=3$

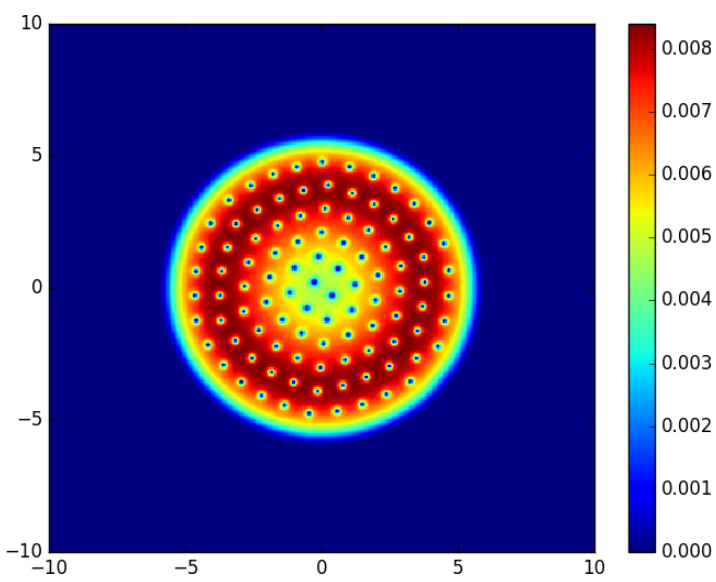

(b) $\beta=10000, \omega=2.5$

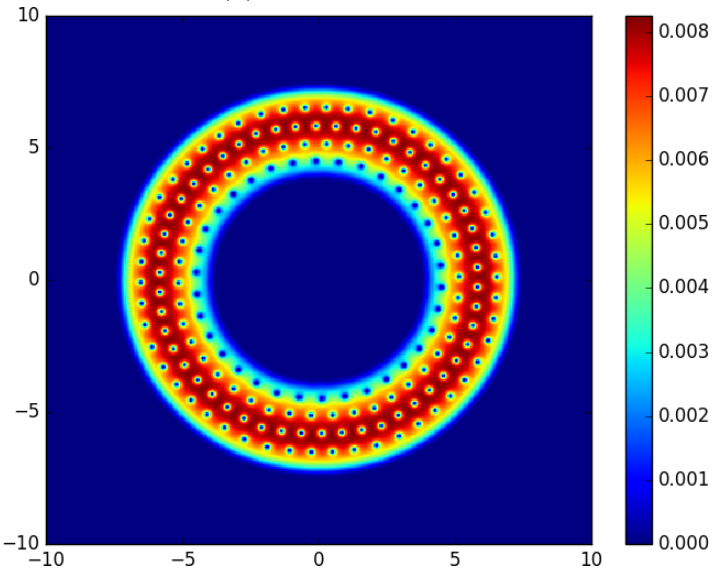

(d) $\beta=10000, \omega=4$

Figure 15: Contour plots of the density function $\left|\phi_{g}\right|^{2}$, for $\beta=10000$ (procs $=32$ ).

\begin{tabular}{|c||c|c|c|c|c|c|c|c|}
\hline$\beta$ & $\omega=1$ & 1.5 & 2 & 2.5 & 3 & 3.5 & 4 & 4.5 \\
\hline 1000 & 57.79 & 53.48 & 91.28 & 123.36 & 132.23 & 173.64 & 158.41 & 357.56 \\
5000 & 97.66 & 387.07 & 352.27 & 685.75 & 329.34 & 254.04 & 253.72 & 4823.42 \\
10000 & 489.68 & 1104.76 & 1279.08 & 562.53 & 960.99 & 1170.01 & 3164.85 & 5603.2 \\
\hline
\end{tabular}

Figure 16: CPUs time (seconds) to compute the ground states of the GPE for various values of $\omega$ and $\beta$ (procs $=32$ ). 


\begin{tabular}{|c|c|r|r|}
\hline & \# it & time (s) & speedup \\
\hline 1 & 951 & 1219.50 & 1.00 \\
2 & 888 & 605.14 & 2.02 \\
4 & 891 & 310.98 & 3.92 \\
8 & 886 & 163.42 & 7.46 \\
16 & 914 & 96.76 & 12.6 \\
32 & 876 & 57.79 & 21.1 \\
\hline
\end{tabular}

(a) $\beta=1000, \omega=1$

\begin{tabular}{|c|r|r|r|}
\hline & \# it & time (s) & speedup \\
\hline 1 & 1518 & 1965.75 & 1.00 \\
2 & 1888 & 1312.84 & 1.50 \\
4 & 1805 & 650.94 & 3.02 \\
8 & 1850 & 344.82 & 5.70 \\
16 & 1791 & 192.15 & 10.23 \\
32 & 1839 & 123.36 & 15.94 \\
\hline
\end{tabular}

(c) $\beta=1000, \omega=2.5$

\begin{tabular}{|c|c|r|r|}
\hline & \# it & time (s) & speedup \\
\hline 1 & 1390 & 1768.86 & 1.00 \\
2 & 1411 & 992.01 & 1.78 \\
4 & 1362 & 488.91 & 3.62 \\
8 & 1378 & 255.94 & 6.91 \\
16 & 1347 & 146.16 & 12.10 \\
32 & 1377 & 91.28 & 19.38 \\
\hline
\end{tabular}

(b) $\beta=1000, \omega=2$

\begin{tabular}{|c|c|r|r|}
\hline & \# it & time (s) & speedup \\
\hline 1 & 7746 & 9976.62 & 1.00 \\
2 & 6434 & 4455.09 & 2.24 \\
4 & 7298 & 2631.25 & 3.79 \\
8 & 7246 & 1355.25 & 7.36 \\
16 & 7292 & 796.05 & 12.53 \\
32 & 7387 & 498.40 & 20.02 \\
\hline
\end{tabular}

(d) $\beta=1000, \omega=5$

Figure 17: 2D scalability tests.

\begin{tabular}{|c|c|r|c|r|}
\hline & \# it & \multicolumn{2}{|c|}{ time } & speedup \\
\hline 32 & 18105 & $10971.45 \mathrm{~s}$ & $3 \mathrm{hrs}$ & 1.0 \\
128 & 18560 & $3973.70 \mathrm{~s}$ & $66 \mathrm{~min}$ & 2.76 \\
\hline
\end{tabular}

Figure 18: Ground states of the GPE for $\beta=10000, \omega=5, M=2048$.

\section{Conclusion}

In this paper, we presented BEC2HPC which is a parallel solver for computing the stationary states of the rotating Gross-Pitaevskii equation for the modelling of 2D/3D Bose-Einstein condensates. The scheme implemented in BEC2HPC is based on a preconditioned conjugate gradient for the minimization of the energy functional under normalization constraint, combined with a pseudo-spectral approximation scheme in space (using FFT). This leads to an efficient and robust code for complex problems, that can also be used for problems related to the nonlinear Schrödinger equation. After a presentation of the implementation aspects, we explain how to use the code on a first 2D example. More complicate 2D and 3D test cases are presented next to illustrate some specific coding aspects of the code and to show the scalability of the code for larger problems.

Future developments of BEC2HPC concern the possibility of simulating the dynamics of the rotating GPE by various schemes, the extension to systems of GPE (stationary states and dynamics) and the possibility to simulate nonlocal nonlinear effects like for example for the case of dipole-dipole interactions.

\section{Acknowledgements}

The authors acknowledge the support from the Inria associate team BEC2HPC (Bose-Einstein Condensates: Computation and HPC simulation (https://team.inria.fr/bec2hpc/)). Q. Tang also acknowledge the support from the National Natural Science Foundation of China (No. 11971335). 


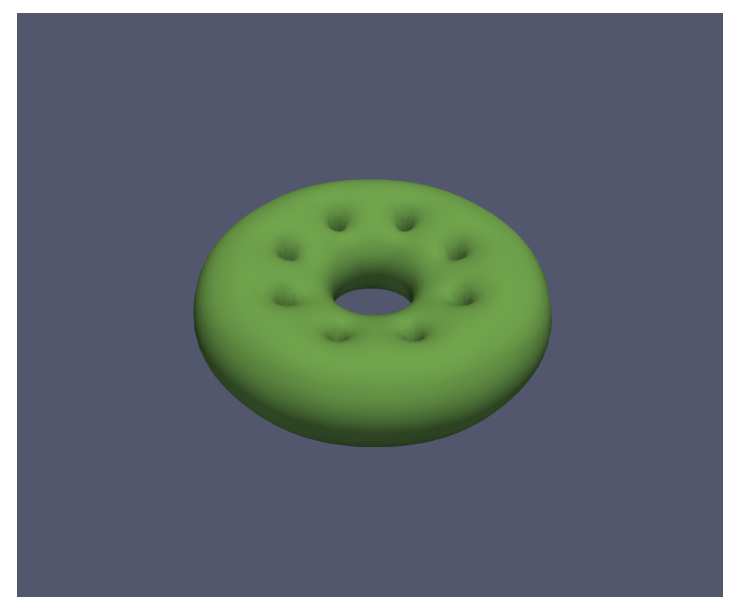

(a) $\beta=100, \omega=1.4$

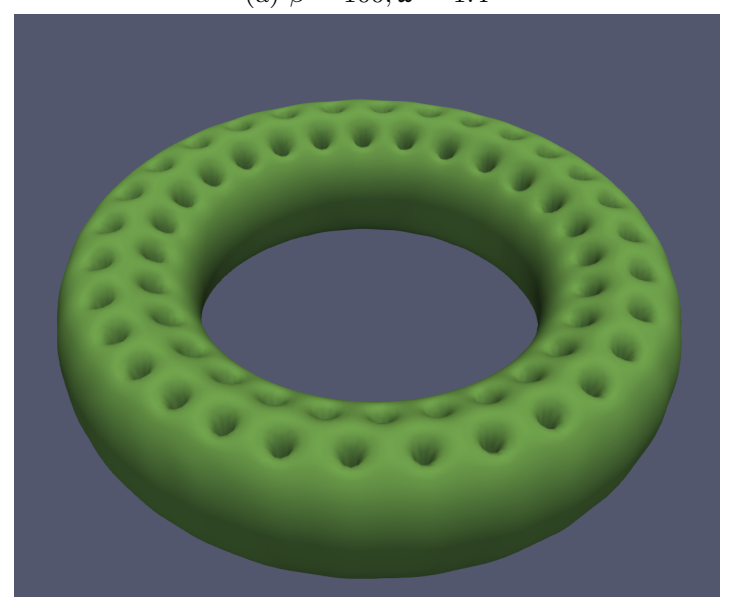

(c) $\beta=5000, \omega=3$

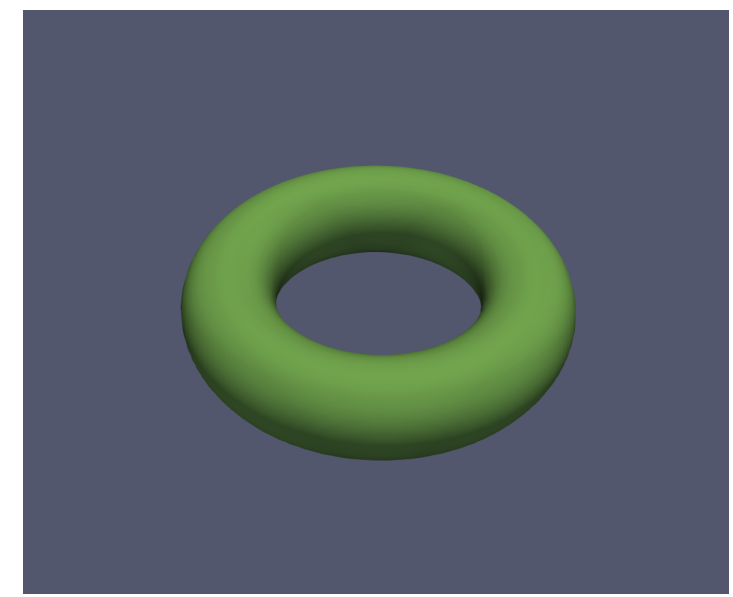

(b) $\beta=100, \omega=1.8$

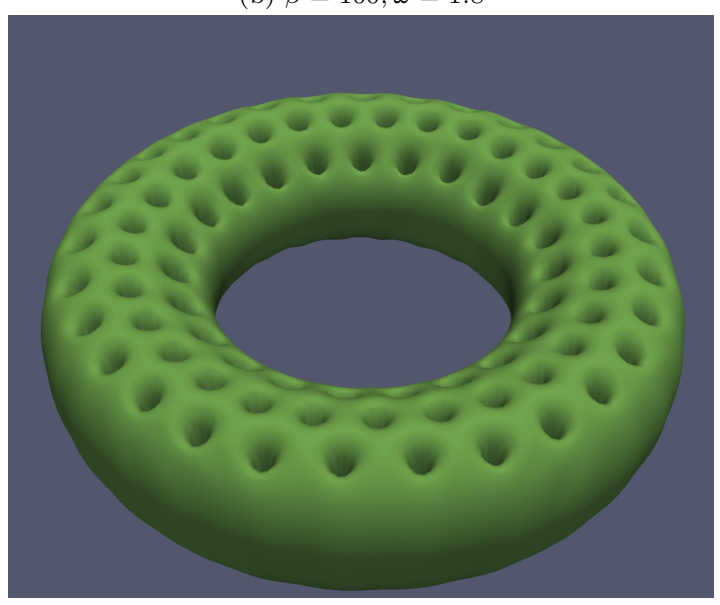

(d) $\beta=10000, \omega=3$

Figure 19: Isosurface $\left|\phi_{g}\right|^{2}=10^{-3}$.

\begin{tabular}{|c|c|r|r|}
\hline & \# it & time (s) & speedup \\
\hline 4 & 753 & 1479.90 & 1.00 \\
8 & 756 & 780.97 & 1.89 \\
16 & 755 & 437.01 & 3.39 \\
32 & 755 & 264.64 & 5.59 \\
\hline
\end{tabular}

(a) $\beta=100, \omega=1.4$

\begin{tabular}{|c|c|c|r|}
\hline & \# it & time (s) & speedup \\
\hline 4 & 3632 & 7019.09 & 1.00 \\
8 & 3713 & 3801.36 & 1.85 \\
16 & 3907 & 2263.74 & 3.1 \\
32 & 4257 & 2409.29 & 2.91 \\
\hline
\end{tabular}

(c) $\beta=5000, \omega=3$

\begin{tabular}{|c|c|r|r|}
\hline & \# it & time (s) & speedup \\
\hline 4 & 514 & 1057.14 & 1.00 \\
8 & 515 & 537.19 & 1.97 \\
16 & 515 & 292.62 & 3.61 \\
32 & 517 & 177.69 & 5.95 \\
\hline
\end{tabular}

(b) $\beta=100, \omega=1.8$

\begin{tabular}{|c|r|r|r|}
\hline & \# it & time $(\mathrm{s})$ & speedup \\
\hline 4 & 6512 & 13091.23 & 1.00 \\
8 & 6602 & 6843.93 & 1.91 \\
16 & 7450 & 4352.26 & 3.01 \\
32 & 7656 & 3411.37 & 3.84 \\
\hline
\end{tabular}

(d) $\beta=10000, \omega=3$

Figure 20: 3D scalability tests $(M=128)$. The computational domain is $[-8,8]^{3}, h=\frac{1}{8}(M=128), \varepsilon=10^{-12}$. 


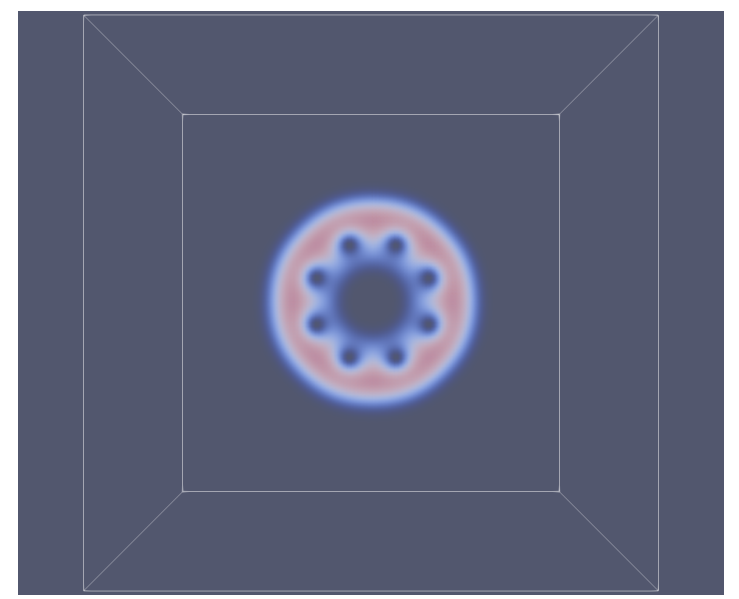

(a) $\beta=100, \omega=1.4$

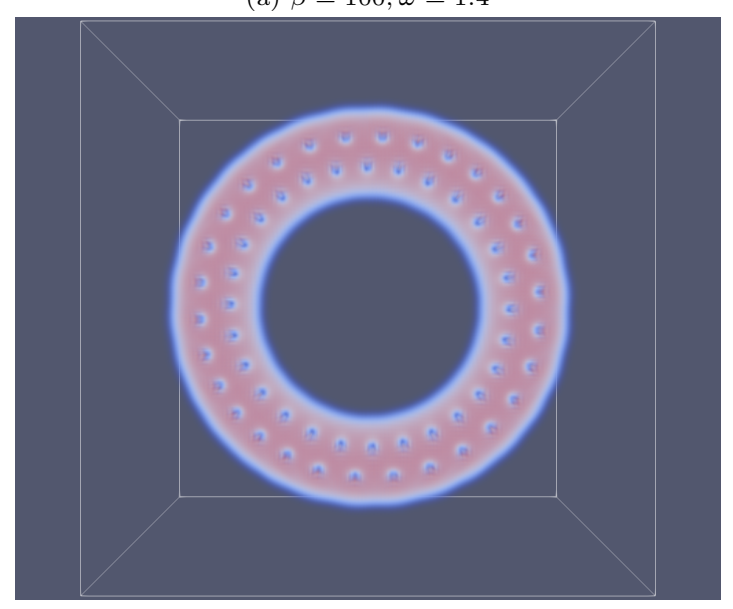

(c) $\beta=5000, \omega=3$

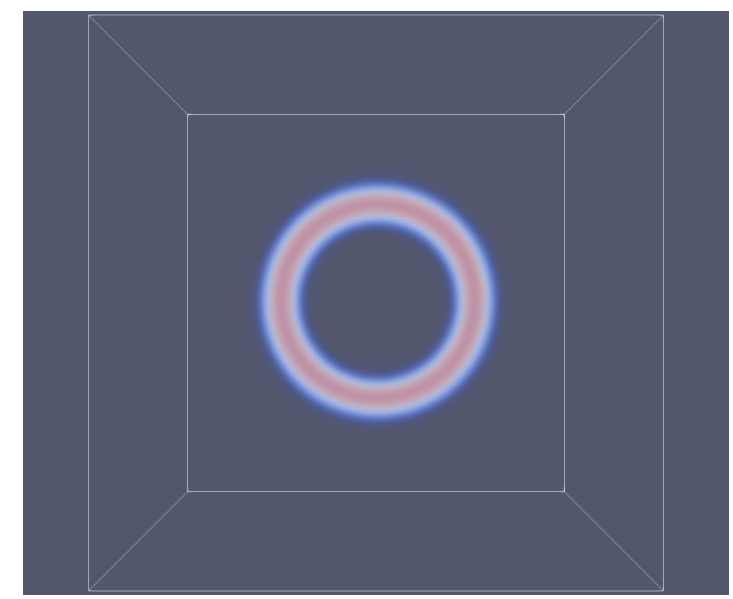

(b) $\beta=100, \omega=1.8$

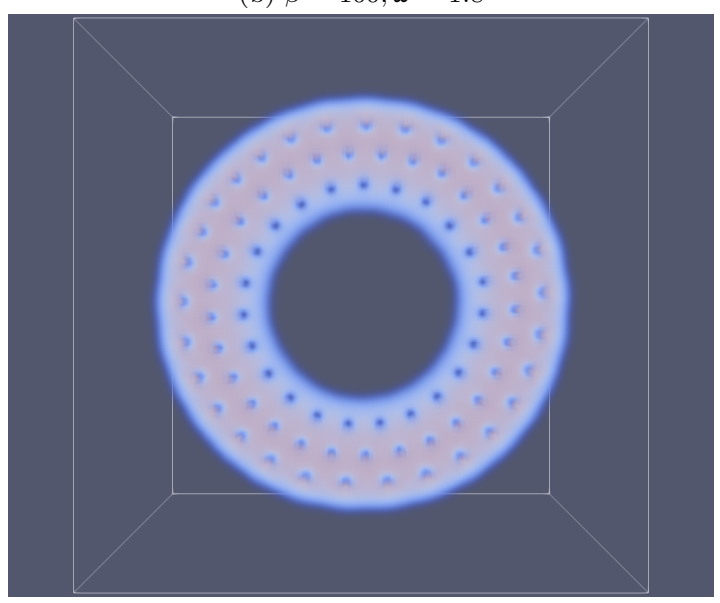

(d) $\beta=10000, \omega=3$

Figure 21: 3D color map of $\left|\phi_{g}\right|^{2}$.

\begin{tabular}{|c|c|r|r|}
\hline & \# it & time (s) & speedup \\
\hline 32 & 1609 & 4541.32 & 1.00 \\
64 & 1617 & 2731.90 & 1.66 \\
128 & 1611 & 2173.89 & 2.09 \\
256 & 1611 & 839.60 & 5.41 \\
\hline
\end{tabular}

(a) $\beta=100, \omega=1.4$

\begin{tabular}{|c|r|r|r|}
\multicolumn{5}{c}{ (a) $\beta=100, \omega=1.4$} \\
\hline 32 & \# it & time (s) & speedup \\
64 & 4071 & 11158.37 & 1.00 \\
128 & 4330 & 5170.47 & 1.56 \\
256 & 4340 & 2335.89 & 4.78 \\
\hline
\end{tabular}

(c) $\beta=5000, \omega=3$

\begin{tabular}{|c|c|r|r|}
\hline & \# it & time (s) & speedup \\
\hline 32 & 1986 & 5404.94 & 1.00 \\
64 & 2025 & 3300.06 & 1.64 \\
128 & 2028 & 2366.42 & 2.28 \\
256 & 2035 & 1215.22 & 4.45 \\
\hline
\end{tabular}

(b) $\beta=100, \omega=1.8$

\begin{tabular}{|c|c|c|r|}
\hline & \# it & time (s) & speedup \\
\hline 256 & 22075 & 12153.14 & 1.0 \\
\hline
\end{tabular}

(d) $\beta=10000, \omega=3$

Figure 22: 3D scalability tests $(M=256)$. Domain is $[-8,8]^{3}, h=\frac{1}{16}(M=256), \varepsilon=10^{-12}$. 


\section{References}

[1] J.R. Abo-Shaeer, C. Raman, J.M. Vogels, and W. Ketterle, Observation of vortex lattices in Bose-Einstein condensates, Science 292 (2001), no. 5516, 476-479.

[2] P-A Absil, R. Mahony, and R. Sepulchre, Optimization Algorithms on Matrix Manifolds, Princeton University Press, 2009.

[3] S.K. Adhikari, Numerical solution of the two-dimensional Gross-Pitaevskii equation for trapped interacting atoms, Physics Letters A 265 (2000), no. 1-2, 91-96.

[4] J. Ahrens, B. Geveci, and C. Law, Paraview: An end-user tool for large data visualization, The visualization handbook 717 (2005).

[5] M.H. Anderson, J.R. Ensher, M.R. Matthews, C.E. Wieman, and E.A. Cornell, Observation of Bose-Einstein condensation in a dilute atomic vapor, Science 269 (1995), no. 5221, 198-201.

[6] X. Antoine, W. Bao, and C. Besse, Computational methods for the dynamics of the nonlinear Schrödinger/GrossPitaevskii equations, Computer Physics Communications 184 (2013), no. 12, 2621-2633.

[7] X. Antoine and R. Duboscq, GPELab, a Matlab toolbox to solve Gross-Pitaevskii equations I: Computation of stationary solutions, Computer Physics Communications 185 (2014), no. 11, 2969-2991.

[8] _ Robust and efficient preconditioned Krylov spectral solvers for computing the ground states of fast rotating and strongly interacting Bose-Einstein condensates, Journal of Computational Physics 258 (2014), $509-523$.

[9] - GPELab, a Matlab toolbox to solve Gross-Pitaevskii equations II: Dynamics and stochastic simulations, Computer Physics Communications 193 (2015), 95-117.

[10] - Modeling and Computation of Bose-Einstein Condensates: Stationary States, Nucleation, Dynamics, Stochasticity, Nonlinear Optical and Atomic Systems: at the Interface of Physics and Mathematics (Besse, C and Garreau, JC, ed.), Lecture Notes in Mathematics, vol. 2146, 2015, pp. 49-145.

[11] X. Antoine, A. Levitt, and Q. Tang, Efficient spectral computation of the stationary states of rotating Bose-Einstein condensates by preconditioned nonlinear conjugate gradient methods, Journal of Computational Physics $\mathbf{3 4 3}$ (2017), $92-109$.

[12] X. Antoine, Q. Tang, and J. Zhang, On the numerical solution and dynamical laws of nonlinear fractional Schrödinger/Gross-Pitaevskii equations, International Journal of Computer Mathematics 95 (2018), no. 6-7, 14231443.

[13] X. Antoine, Q. Tang, and Y. Zhang, On the ground states and dynamics of space fractional nonlinear Schrödinger/Gross-Pitaevskii equations with rotation term and nonlocal nonlinear interactions, Journal of Computational Physics 325 (2016), 74-97.

[14] _ A preconditioned conjugated gradient method for computing ground states of rotating dipolar Bose-Einstein condensates via kernel truncation method for dipole-dipole interaction evaluation, Communications in Computational Physics 24 (2018), no. 4, 966-988.

[15] W. Bao, Ground states and dynamics of multi-component Bose-Einstein condensates, Multiscale Modeling and Simulation: A SIAM Interdisciplinary Journal 2 (2004), no. 2, 210-236.

[16] W. Bao and Y. Cai, Ground states of two-component Bose-Einstein condensates with an internal atomic Josephson junction, East Asian Journal on Applied Mathematics 1 (2011), 49-81.

[17] Mathematical theory and numerical methods for Bose-Einstein condensation, Kinetic and Related Models 6 (2013), no. 1, 1-135.

[18] W. Bao, Y. Cai, and H. Wang, Efficient numerical methods for computing ground states and dynamics of dipolar Bose-Einstein condensates, Journal of Computational Physics 229 (2010), no. 20, 7874-7892.

[19] W. Bao and Q. Du, Computing the ground state solution of Bose-Einstein condensates by a normalized gradient flow, SIAM Journal on Scientific Computing 25 (2004), no. 5, 1674-1697.

[20] W. Bao, S. Jiang, Q. Tang, and Y. Zhang, Computing the ground state and dynamics of the nonlinear Schrödinger equation with nonlocal interactions via the nonuniform FFT, Journal of Computational Physics 296 (2015), $72-89$.

[21] W. Bao and W. Tang, Ground-state solution of Bose-Einstein condensate by directly minimizing the energy functional, Journal of Computational Physics 187 (2003), no. 1, 230-254.

[22] D. Baye and J.M. Sparenberg, Resolution of the Gross-Pitaevskii equation with the imaginary-time method on a Lagrange mesh, Physical Review E 82 (2010), no. 5.

[23] C.C. Bradley, C.A. Sackett, J.J. Tollett, and R.G. Hulet, Evidence of Bose-Einstein condensation in an atomic gas with attractive interactions, Physical Review Letters 75 (1995), no. 9, 1687-1690.

[24] V. Bretin, S. Stock, Y. Seurin, and J. Dalibard, Fast rotation of a Bose-Einstein condensate, Physical Review Letters 92 (2004), no. 5

[25] T. Byrnes, K. Wen, and Y. Yamamoto, Macroscopic quantum computation using Bose-Einstein condensates, Physical Review A 85 (2012), no. 4.

[26] M. Caliari, A. Ostermann, S. Rainer, and M. Thalhammer, A minimisation approach for computing the ground state of Gross-Pitaevskii systems, Journal of Computational Physics 228 (2009), no. 2, 349-360.

[27] R.M. Caplan, NLSEmagic: Nonlinear Schrödinger equation multi-dimensional Matlab-based GPU-accelerated integrators using compact high-order schemes, Computer Physics Communications 184 (2013), no. 4, 1250-1271.

[28] M.M. Cerimele, M.L. Chiofalo, F. Pistella, S. Succi, and M.P. Tosi, Numerical solution of the Gross-Pitaevskii equation using an explicit finite-difference scheme: An application to trapped Bose-Einstein condensates, Physical Review E 62 (2000), no. 1, 1382-1389.

[29] M.L. Chiofalo, S. Succi, and M.P. Tosi, Ground state of trapped interacting Bose-Einstein condensates by an explicit imaginary-time algorithm, Physical Review E 62 (2000), no. 5, 7438-7444.

[30] F. Dalfovo, S. Giorgini, L.P. Pitaevskii, and S. Stringari, Theory of Bose-Einstein condensation in trapped gases, Review of Modern Physics 71 (1999), no. 3, 463-512.

[31] I. Danaila and F. Hecht, A finite element method with mesh adaptivity for computing vortex states in fast-rotating Bose-Einstein condensates, Journal of Computational Physics 229 (2010), no. 19, 6946-6960.

[32] I. Danaila and P. Kazemi, A new Sobolev gradient method for direct minimization of the Gross-Pitaevskii energy with rotation, SIAM Journal on Scientific Computing 32 (2010), no. 5, 2447-2467. 
[33] I. Danaila and B. Protas, Computation of ground states of the Gross-Pitaevskii functional via Riemannian optimization, SIAM Journal on Scientific Computing 39 (2017), no. 6, B1102-B1129.

[34] K.B. David, M.O. Mewes, M.R. Andrews, N.J. Vandruten, D.S. Durfee, D.M. Kurn, and W. Ketterle, Bose-Einstein Condensation in gas of sodium atoms, Physical Review Letters 75 (1995), no. 22, 3969-3973.

[35] C.M. Dion and E. Cances, Ground state of the time-independent Gross-Pitaevskii equation, Computer Physics Communications 177 (2007), no. 10, 787-798.

[36] A. Edelman, T. A Arias, and S.T. Smith, The geometry of algorithms with orthogonality constraints, SIAM Journal on Matrix Analysis and Applications 20 (1998), no. 2, 303-353.

[37] The Epetra Project Team, The Epetra Project Website, https://trilinos.github.io/epetra.html, 2020.

[38] A. L. Fetter, B. Jackson, and S. Stringari, Rapid rotation of a Bose-Einstein condensate in a harmonic plus quartic trap, Physical Review A $\mathbf{7 1}$ (2005), 013605.

[39] M. Frigo and S. G. Johnson, The design and implementation of FFTW3, Proceedings of the IEEE 93 (2005), no. 2, 216-231, Special issue on "Program Generation, Optimization, and Platform Adaptation".

[40] HDF Group et al., HDF5 User's Guide, http://www.hdfgroup.org/HDF5, 2012.

[41] HashiCorp, Vagrant, https://www.vagrantup.com/, 2010-2010.

[42] U. Hohenester, OCTBEC, a Matlab toolbox for optimal quantum control of Bose-Einstein condensates, Computer Physics Communications 185 (2014), no. 1, $194-216$.

[43] B.-W. Jeng, Y.-S. Wang, and C.-S. Chien, A two-parameter continuation algorithm for vortex pinning in rotating Bose-Einstein condensates, Computer Physics Communications 184 (2013), no. 3, 493 -508.

[44] R. Kishor Kumar, V. Lončar, P. Muruganandam, S.K. Adhikari, and A. Balaž, $C$ and Fortran OpenMP programs for rotating Bose-Einstein condensates, Computer Physics Communications 240 (2019), 74-82.

[45] R.K. Kumar, L.E. Young-S, D. Vudragović, A. Balaž, and P. Muruganandam, Fortran and C programs for the timedependent dipolar Gross-Pitaevskii equation in an anisotropic trap, Computer Physics Communications 195 (2015), no. $9,117-128$.

[46] N. Li and S. Laizet, 2DECOMP \& FFT - A Highly Scalable 2D Decomposition Library and FFT Interface, 2010.

[47] V. Lončar, L.E. Young-S, P. Muruganandam, S.K. Adhikari, and A. Balaž, OpenMP, OpenMP/MPI, and CUDA/MPI $C$ programs for solving the time-dependent dipolar Gross-Pitaevskii equation, Computer Physics Communications 209 (2016), 190-196.

[48] K.W. Madison, F. Chevy, V. Bretin, and J. Dalibard, Stationary states of a rotating Bose-Einstein condensate: Routes to vortex nucleation, Physical Review Letters 86 (2001), no. 20, 4443-4446.

[49] K.W. Madison, F. Chevy, W. Wohlleben, and J. Dalibard, Vortex formation in a stirred Bose-Einstein condensate, Physical Review Letters 84 (2000), no. 5, 806-809.

[50] Z. Marojević, E. Göklö, and C. Lämmerzahl, ATUS-PRO: A FEM-based solver for the time-dependent and stationary Gross-Pitaevskii equation, Computer Physics Communications 202 (2016), 216-232.

[51] M.R. Matthews, B.P. Anderson, P.C. Haljan, D.S. Hall, C.E. Wieman, and E.A. Cornell, Vortices in a Bose-Einstein condensate, Physical Review Letters 83 (1999), no. 13, 2498-2501.

[52] P. Muruganandam and S.K. Adhikari, Fortran programs for the time-dependent Gross-Pitaevskii equation in a fully anisotropic trap, Computer Physics Communications 180 (2009), no. 10, 1888-1912.

[53] D. Pekurovsky, P3DFFT: a framework for parallel computations of Fourier transforms in three dimensions, CoRR abs/1905.02803 (2019).

[54] M. Pippig, PFFT: An Extension of FFTW to Massively Parallel Architectures, SIAM Journal on Scientific Computing 35 (2013), no. 3, C213-C236.

[55] C. Raman, J.R. Abo-Shaeer, J.M. Vogels, K. Xu, and W. Ketterle, Vortex nucleation in a stirred Bose-Einstein condensate, Physical Review Letters 87 (2001), no. 21.

[56] B. Satarič, V. Slavnič, A. Balač A. Beličc, P. Muruganandam, and S.K. Adhikari, Hybrid OpenMP/MPI programs for solving the time-dependent Gross-Pitaevskii equation in a fully anisotropic trap, Computer Physics Communications 200 (2016), 411-417.

[57] G. Vergez, I. Danaila, S. Auliac, and F. Hecht, A finite-element toolbox for the stationary Gross-Pitaevskii equation with rotation, Computer Physics Communications 209 (2016), 144-162.

[58] D. Vudragović, I. Vidanović, A. Balaž, P. Muruganandam, and S.K. Adhikari, C programs for solving the timedependent Gross-Pitaevskii equation in a fully anisotropic trap, Computer Physics Communications 183 (2012), no. 9, 2021-2025.

[59] Y.-S. Wang and C.-S. Chien, A spectral-Galerkin continuation method using Chebyshev polynomials for the numerical solutions of the Gross-Pitaevskii equation, Journal of Computational and Applied Mathematics 235 (2011), no. 8, $2740-2757$.

[60] Y.-S. Wang, B.-W. Jeng, and C.-S. Chien, A two-parameter continuation method for rotating two-component BoseEinstein condensates in optical lattices, Communications in Computational Physics 13 (2013), 442-460.

[61] X. Wu, Z. Wen, and W. Bao, A regularized Newton method for computing ground states of Bose-Einstein condensates, Journal of Scientific Computing 73 (2017), 303-329.

[62] L.E. Young-S, P. Muruganandam, S.K. Adhikari, V. Lončar, D. Vudragović, and A. Balaž, OpenMP GNU and Intel Fortran programs for solving the time-dependent Gross-Pitaevskii equation, Computer Physics Communications 220 (2017), 503-506.

[63] C. Yuce and Z. Oztas, Off-axis vortex in a rotating dipolar Bose-Einstein condensate, Journal of Physics B-Atomic Molecular and Optical Physics 43 (2010), no. 13.

[64] R. Zeng and Y. Zhang, Efficiently computing vortex lattices in rapid rotating Bose-Einstein condensates, Computer Physics Communications 180 (2009), no. 6, 854-860. 